\title{
AN AFFINE FRAMEWORK FOR ANALYTICAL MECHANICS
}

\author{
PAWEŁ URBAŃSKI \\ Division of Mathematical Methods in Physics, University of Warsaw \\ Hoża 74, 00-682 Warszawa, Poland \\ E-mail: urbanski@fuw.edu.pl
}

\begin{abstract}
An affine Cartan calculus is developed. The concepts of special affine bundles and special affine duality are introduced. The canonical isomorphisms, fundamental for Lagrangian and Hamiltonian formulations of the dynamics in the affine setting are proved.

1. Introduction. Gauge independence of the Langrangian formulation of dynamics of charged particles can be achieved by increasing the dimension of the configuration space of the particle ([8]). The four dimensional space-time of general relativity is replaced by the five dimensional space-time-phase of Kaluza. The phase space of the particle is the cotangent bundle of the Kaluza space and the gauge independent Lagrangian is a function on the tangent bundle of the Kaluza space. A similar construction makes possible a frame-independent formulation of the Newtonian analytical mechanics (see [13] for details).

There is an alternate approach, based on ideas and results of Tulczyjew, in which the four dimensional space-time is used as the configuration space of the charged particle $([14])$. The phase space is no longer a cotangent bundle and not even a vector bundle. It is an affine bundle modeled on the cotangent bundle $\mathrm{T}^{*} M$ of the space-time manifold $M$. The dynamics is a generalized Hamiltonian system, but the Lagrangian generating object is not a function. It is a section of an affine bundle modeled on $\mathrm{T} M \times \mathbb{R}$.

Also time-dependent mechanics in the inhomogeneous formulation requires affine objects. Here infinitesimal configurations are first jets of motions interpreted as sections of the space-time fibered over time, and are elements of an affine bundle over the space-time. The phase manifold is a vector bundle, however the Hamiltonian is not a function but a section of a bundle over the phase manifold ([16]).
\end{abstract}

2000 Mathematics Subject Classification: 70G45, 70H03, 58A99.

Supported by KBN, grant No 2 PO3A 04118.

The paper is in final form and no version of it will be published elsewhere. 
We develop the geometric framework for these approaches. The standard geometric constructions based on the algebra of functions on a manifold $M$ are replaced by constructions based on the affine space of sections of an affine bundle $\zeta: Z \rightarrow M$, modeled on the trivial bundle $M \times \mathbb{R}$.

In Section 2 we adapt the definition of a covector as an equivalence class of functions to the affine case. The manifold $\mathrm{P} Z$ of affine covectors is an affine bundle modeled on $\mathrm{T}^{*} M$ and carries a canonical symplectic structure. Sections of $\zeta$ generate Lagrangian submanifolds of $\mathrm{P} Z$.

In analytical mechanics of a particle the phase space is considered a vector bundle, dual to the vector bundle of infinitesimal configurations (velocities). In the presented approach vector bundles are replaced by affine bundles, vector spaces by affine spaces. Affine functions on an affine space $\underline{A}$ are replaced by affine sections of a bundle $\zeta_{A}: A \rightarrow \underline{A}$ in the category of affine spaces, modeled on $\underline{A} \times \mathbb{R}$. This means that $A, \underline{A}$ are affine spaces and $\zeta_{A}$ is an affine surjective mapping. Such a bundle is uniquely determined be the affine space $A$ and a distinguished vector $v_{1}$ in the model vector space $\mathrm{V}(A)$. We call it a special affine space (Section 3).

The dual object to a special affine space $\boldsymbol{A}=\left(A, v_{1}\right)$ is the set of affine sections of $\zeta_{A}$. It carries a natural structure of an affine space with the vector space of affine functions on $\underline{A}$ as the model vector space. With the constant function 1 the dual affine space becomes a special affine space. We introduce in an obvious way the concept of a special affine bundle. Principal examples of special affine bundles are $\mathrm{P} Z \times \mathbb{R} \rightarrow M$, the first jet (contact) bundle $\mathrm{C} Z \rightarrow M$, and their duals.

It is convenient to represent an element of the dual object as a morphism in the category. For this purpose we identify a section $\sigma$ of $\zeta_{A}$ with a morphism $\widetilde{\varphi}_{\sigma}: \boldsymbol{A} \rightarrow \boldsymbol{I}$, where $\boldsymbol{I}=(\mathbb{R}, 1)$ and $\widetilde{\varphi}(a)=a-\varphi\left(\zeta_{A}(a)\right)$. We must be aware of the fact that with this representation the canonical pairing is not symmetric, but skew-symmetric.

The theory of special affine spaces (bundles) and duality is developed in Sections 3, 4 and 5 . In Section 3 we present the main algebraic constructions in the category of special affine spaces. In Section 4 we interpret the bundles dual to $\mathrm{P} Z \times \mathbb{R}$ and $C Z$ in terms of the tangent bundle T $Z$. In Section 5 we prove the existence of canonical isomorphisms between $\boldsymbol{C} \boldsymbol{A}$ and $\boldsymbol{C A}^{\#}$, where $\boldsymbol{A}$ is a special affine bundle and $\boldsymbol{A}^{\#}$ is the special affine dual bundle. This isomorphism corresponds to the canonical isomorphism between $\mathrm{T}^{*} E$ and $\mathrm{T}^{*} E^{*}$ for a vector bundle $E$, and makes possible the Legendre transformation.

The Lagrange formulation of the dynamics of a particle is possible because of the canonical Tulczyjew isomorphism (symplectomorphism) of $\mathrm{TT}^{*} M$ and $\mathrm{T}^{*} \mathrm{~T} M$. In Section 5 we give a proof of an affine counterpart for this isomorphism. As a consequence we obtain an affine setting for the Lagrangian formulation of the dynamics. As an example we discuss the case of a relativistic charged particle (Section 6).

A similar approach to time-dependent non-relativistic mechanics has been recently developed be E. Massa with collaborators $([6,7,17])$, W. Sarlet, and E. Martínez ([5, $10,11])$.

This work is a contribution to a programme of study of geometric foundations of physical theories conducted jointly with Professor Tulczyjew at the University of Camerino. 


\section{Affine phase and contact spaces}

2.1. Affine spaces and affine bundles. An affine space is a triple $(A, V, \alpha)$, where $A$ is a set, $V$ is a real vector space of finite dimension and $\alpha$ is a mapping $\alpha: A \times A \rightarrow V$ such that

(1) $\alpha\left(a_{3}, a_{2}\right)+\alpha\left(a_{2}, a_{1}\right)+\alpha\left(a_{1}, a_{3}\right)=0$;

(2) the mapping $\alpha(\cdot, a): A \rightarrow V$ is bijective for each $a \in A$.

We shall also write simply $A$ to denote the affine space $(A, V, \alpha)$ and $\mathrm{V}(A)$ to denote $V$. If $(A, V, \alpha)$ then also $(A, V,-\alpha)$ is an affine space. We will write for brevity $\bar{A}$ to denote the affine space $(A, V,-\alpha)$. We will write also $a_{2}-a_{1}$ instead of $\alpha\left(a_{2}, a_{1}\right)$ and we will denote by $a+v$ the unique point $a^{\prime} \in A$ such that $a^{\prime}-a=v$.

Let $\xi: E \rightarrow M$ be a vector bundle. An affine bundle modeled on $\xi$ is a differential fibration $\eta: A \rightarrow M$ and a differentiable mapping $\rho: A \times_{M} A \rightarrow E$ such that

(1) $\xi \circ \rho=\eta \times_{M} \eta$,

(2) $\rho\left(a_{3}, a_{2}\right)+\rho\left(a_{2}, a_{1}\right)=\rho\left(a_{3}, a_{1}\right)$ for each triple $\left(a_{3}, a_{2}, a_{1}\right) \in A \times_{M} A \times_{M} A$,

(3) for each local section $\sigma: U \rightarrow A$ of $\eta$, the mapping $\sigma_{0}: \rightarrow E$ defined by $\sigma_{0}(m)=$ $\rho(\sigma(m), \sigma(m))$ is the zero section of $\xi$ over $U$,

(4) for each local section $\sigma: U \rightarrow A$ of $\eta$, the mapping $\rho_{\sigma}: \eta^{-1}(U) \rightarrow \xi^{-1}(U)$ defined by $\rho_{\sigma}(a)=\rho(a, \sigma(\eta(a)))$ is a diffeomorphism.

We will write simply $A$ to denote the affine bundle $(A, E, \rho)$ and $\mathrm{V}(A)$ to denote $E$.

Remark. Any section $\sigma \in \operatorname{Sec}(\eta)$ of $A$ induces an obvious isomorphism $I_{\sigma} \in$ $\operatorname{Aff}_{M}(A, \mathrm{~V}(A))$ of affine bundles:

$$
A_{m} \ni a \mapsto I_{\sigma}(a)=a-\sigma(m) \in \mathrm{V}\left(A_{m}\right) .
$$

Let $\tau_{i}: A_{i} \rightarrow M$ be an affine bundle modeled on a vector bundle $\mathrm{v}\left(\tau_{i}\right): \mathrm{V}\left(A_{i}\right) \rightarrow M$, $i=1,2,3$. Note that the space $\mathcal{A}_{i}$ of sections of $\tau_{i}$ is an affine space modeled on the space $\mathrm{V}\left(\mathcal{A}_{i}\right)$ of sections of $\mathrm{v}\left(\tau_{i}\right)$. For an affine bundle morphism $\phi: A_{1} \rightarrow A_{2}$ we denote by $\phi_{\mathrm{V}}: \mathrm{V}\left(A_{1}\right) \rightarrow \mathrm{V}\left(A_{2}\right)$ its linear part, i.e.

$$
\phi_{\mathrm{v}}(v)=\phi(a+v)-\phi(a) \text { for } a \in A, v \in \mathrm{V}(A), \quad \tau_{1}(a)=\mathrm{v}\left(\tau_{1}\right)(v) .
$$

We will denote by $\operatorname{Aff}_{M}\left(A_{1}, A_{2}\right)$ (resp. $\operatorname{Hom}_{M}\left(V_{1}, V_{2}\right)$ ) the set of morphisms over the identity on the base in the affine (resp. vector) case. We shall also write $\operatorname{Aff}(A, \mathbb{R})$ instead of $\operatorname{Aff}_{M}(A, M \times \mathbb{R})$ and $\operatorname{Lin}(V, \mathbb{R})$ instead of $\operatorname{Hom}_{M}(V, M \times \mathbb{R})$. For a bi-affine mapping

$$
F: A_{1} \times_{M} A_{2} \rightarrow A_{3}
$$

we denote by $F^{\vee}$ and ${ }^{\vee} F$, respectively, the mappings

$$
F^{\vee}: A_{1} \times_{M} \mathrm{~V}\left(A_{2}\right) \rightarrow \mathrm{V}\left(A_{3}\right), \quad\left(a_{1}, v_{2}\right) \mapsto\left(F\left(a_{1}, \cdot\right)\right)_{\mathrm{v}}\left(v_{2}\right),
$$

and

$$
{ }^{\vee} F: \mathrm{V}\left(A_{1}\right) \times_{M} A_{2} \rightarrow \mathrm{V}\left(A_{3}\right), \quad\left(v_{1}, a_{2}\right) \mapsto\left(F\left(\cdot, a_{2}\right)\right)_{\mathrm{v}}\left(v_{1}\right)
$$

These mappings are, respectively, affine-linear and linear-affine in the obvious sense. By $F_{v}$ we denote the bilinear part of $F$, i.e. 


$$
\begin{aligned}
& F_{\mathrm{v}}: \mathrm{V}\left(A_{1}\right) \times_{M} \mathrm{~V}\left(A_{2}\right) \rightarrow \mathrm{V}\left(A_{3}\right), \\
& \quad\left(v_{1}, v_{2}\right) \mapsto\left(F^{\vee}\left(\cdot, v_{2}\right)\right)_{\mathrm{v}}\left(v_{1}\right)=\left({ }^{\mathrm{v}} F\left(v_{1}, \cdot\right)\right)_{\mathrm{v}}\left(v_{2}\right) \\
& \quad=F\left(a_{1}+v_{1}, a_{2}+v_{2}\right)-F\left(a_{1}+v_{2}, a_{2}\right)+F\left(a_{1}, a_{2}\right)-F\left(a_{1}, a_{2}+v_{2}\right)
\end{aligned}
$$

2.2. Affine Cartan calculus. The standard Cartan calculus of differential forms is based on the algebra of differentiable functions on a manifold $M$. In the affine Cartan calculus we replace functions by sections of an affine bundle $\zeta: Z \rightarrow M$ modeled on the trivial bundle $M \times \mathbb{R}$. We can consider $Z$ a principal bundle with the structure group $(\mathbb{R},+)(15)$. The space of sections of $\zeta$ is an affine space modeled on the space of sections of $M \times \mathbb{R}$ and a section of $M \times \mathbb{R}$ we identify with a function on $M$.

2.3. The phase and contact fibrations. Let $\zeta: Z \rightarrow M$ be an affine fibration modeled on the trivial fibration ${ } r_{M}: M \times \mathbb{R} \rightarrow M$. We define an equivalence relation in the set of all pairs $(m, \sigma)$, where $m$ is a point in $M$ and $\sigma$ is a section of $\zeta$. Two pairs $(m, \sigma)$ and $\left(m^{\prime}, \sigma^{\prime}\right)$ are equivalent if $m^{\prime}=m$ and $\mathrm{d}\left(\sigma^{\prime}-\sigma\right)(m)=0$. We denote by $\mathrm{P} Z$ the set of equivalence classes. The class of $(m, \sigma)$ will be denoted by $\mathrm{d} \sigma(m)$ or by $\mathrm{d}_{m} \sigma$ and will be called the differential of $\sigma$ at $m$. We define the canonical projection

$$
\mathrm{P} \zeta: \mathrm{P} Z \rightarrow M, \quad \mathrm{~d} \sigma(m) \mapsto m .
$$

We define also a mapping

$$
\mathrm{P} \rho: \mathrm{P} Z \times_{M} \mathrm{P} Z \rightarrow \mathrm{T}^{*} M, \quad\left(\mathrm{~d} \sigma_{2}(m), \mathrm{d} \sigma_{1}(m)\right) \mapsto \mathrm{d}\left(\sigma_{2}-\sigma_{1}\right)(m) .
$$

The pair $(\mathrm{P} \zeta, \mathrm{P} \rho)$ makes $\mathrm{P} Z$ an affine fibration modeled on the cotangent fibration $\pi_{M}: \mathrm{T}^{*} M \rightarrow M$. This fibration is called the phase fibration of $\zeta$. A section of $\mathrm{P} \zeta$ will be called an affine 1-form.

Let $\alpha: M \rightarrow \mathrm{P} Z$ be an affine 1 -form and let $\sigma$ be a section of $\zeta$. The differential $\mathrm{d}_{m}(\alpha-\mathrm{d} \sigma)$ does not depend on the choice of $\sigma$ and will be called the differential of $\alpha$ at $m$. We will denote it by $\mathrm{d} \alpha(m)$ or by $\mathrm{d}_{m} \alpha$. The differential of an affine 1 -form is an ordinary 2-form.

REMARK. Let us consider a principal bundle $Z$ with structure group $(\mathbb{R},+)$. A section of $\mathrm{P} Z$ defines a connection in $Z$ and can be interpreted as an affine form of this connection.

We define another equivalence relation in the set of all pairs $(m, \sigma)$. Two pairs $(m, \sigma)$ and $\left(m^{\prime}, \sigma^{\prime}\right)$ are equivalent if $m^{\prime}=m, \sigma(m)=\sigma^{\prime}(m)$, and $\mathrm{d}\left(\sigma^{\prime}-\sigma\right)(m)=0$. We can identify the equivalence class of $(m, \sigma)$ with the first jet of the section $\sigma$ with source point $m$. We denote by $C Z$ the set of equivalence classes. The class of $(m, \sigma)$ will be denoted by $\mathrm{c} \sigma(m)$ or by $\mathrm{c}_{m} \sigma$ and will be called the contact element of $\sigma$ at $m$. We define a mapping $\mathrm{C} \zeta: \mathrm{C} Z \rightarrow M$ by $\mathrm{C} \zeta(\mathrm{c} \sigma(m))=m$ and a mapping

$$
\mathrm{C} \rho: \mathrm{C} Z \times{ }_{M} \mathrm{C} Z \rightarrow \mathrm{T}^{*} M \times \mathbb{R}
$$

by

$$
\mathrm{C} \rho\left(\mathrm{d} \sigma_{2}(m), \mathrm{c} \sigma_{1}(m)\right)=\left(\mathrm{d}\left(\sigma_{2}-\sigma_{1}\right)(m), \sigma_{2}(m)-\sigma_{1}(m)\right) .
$$

The pair $(\mathrm{C} \zeta, \mathrm{C} \rho)$ makes $\mathrm{C} Z$ an affine fibration modeled on the fibration $\gamma_{M}: \mathrm{T}^{*} M \times \mathbb{R} \rightarrow$ $M$. This fibration is called the contact fibration of $\zeta$. We have an obvious isomorphism of affine bundles

$$
\mathrm{C} Z=\mathrm{P} Z \times_{M} Z .
$$


Let $Z$ and $Z^{\prime}$ be special affine bundles modeled on $M \times \mathbb{R}$ and $M^{\prime} \times \mathbb{R}$ respectively. A morphism $\Phi: Z \rightarrow Z^{\prime}$ induces a mapping $\Phi^{*}: \operatorname{Sec}\left(Z^{\prime}\right) \rightarrow \operatorname{Sec}(Z)$. The mapping $\Phi^{*}$ and the relation $\Phi^{-1}$ project to relations $\mathrm{P} \Phi: \mathrm{P} Z^{\prime} \rightarrow \mathrm{P} Z$ and $\mathrm{C} \Phi: \mathrm{C} Z^{\prime} \rightarrow \mathrm{C} Z$.

2.4. Canonical structures on $\mathrm{P} Z$ and $C Z$. We show first that $P Z$ carries a canonical symplectic structure. For a chosen section $\sigma$ of $\zeta$ we have isomorphisms (see (34))

$$
I_{\sigma}: Z \rightarrow M \times \mathbb{R}, \quad I_{\mathrm{d} \sigma}: \mathrm{P} Z \rightarrow \mathrm{T}^{*} M
$$

and for two sections $\sigma, \sigma^{\prime}$ the mapping

$$
I_{\mathrm{d} \sigma^{\prime}} \circ I_{\mathrm{d} \sigma}^{-1}: \mathrm{T}^{*} M \rightarrow \mathrm{T}^{*} M, \quad p \mapsto p+\mathrm{d}\left(\sigma-\sigma^{\prime}\right)\left(\pi_{M}(p)\right),
$$

is a symplectomorphism. It follows that the 2-form $I_{\mathrm{d} \sigma}^{*} \omega_{M}$, where $\omega_{M}$ is the canonical symplectic form on $\mathrm{T}^{*} M$, does not depend on the choice of $\sigma$. We will denote this form by $\omega_{\boldsymbol{Z}}$.

There is a canonical projection

$$
\mu: \mathrm{C} Z \rightarrow Z
$$

which is a morphism of affine bundles $\zeta_{\mathrm{C} Z}: \mathrm{C} Z \rightarrow \mathrm{P} Z$ and $\zeta: Z \rightarrow M$, so we have a pullback of sections of $\zeta$ to sections of $\zeta_{\mathrm{C} \boldsymbol{Z}}$. Now we can define a section $\theta_{\boldsymbol{Z}}$ of $\mathrm{P} \zeta_{\mathrm{C} \boldsymbol{Z}}$ : $\mathrm{PC} Z \rightarrow$ $\mathrm{P} Z$ by

$$
\theta_{\boldsymbol{Z}}(p)=\mathrm{d}_{p} \mu^{*} \sigma_{p}
$$

where $\sigma_{p}$ is a section of $\zeta$ which represents $p \in \mathrm{P} Z$. It is easy to see that the canonical symplectic form on $\mathrm{P} Z$ is equal to $\mathrm{d} \theta_{\boldsymbol{Z}}$. The affine 1 -form $\theta_{\boldsymbol{Z}}$ is called the Liouville affine form of $C Z$ and defines the canonical contact structure of $C Z$.

2.5. Generating objects. As in the cotangent bundle, the image of an affine 1-form $\alpha: M \rightarrow \mathrm{P} Z$ is a Lagrangian submanifold of $\left(\mathrm{P} Z, \omega_{\boldsymbol{Z}}\right)$ if and only if it is closed, i.e. if $\mathrm{d} \alpha=0$. If $\alpha=\mathrm{d} \sigma$ for a section $\sigma$ of $Z$ then we say that $\sigma$ is a generating section of the Lagrangian submanifold $\alpha(M)$.

Now, let $C \subset M$ be a submanifold and $\sigma: C \rightarrow Z$ a section of $\zeta$ over $C$. We define a submanifold $L \subset \mathrm{P} Z$ by

$$
L=\left\{p \in \mathrm{P} Z: p=\mathrm{d}_{m} \sigma^{\prime} \text { where } m \in C \text { and } \sigma^{\prime} \mid C=\sigma\right\} .
$$

As in the trivial case, $L$ is a Lagrangian submanifold of $\left(\mathrm{P} Z, \omega_{\boldsymbol{Z}}\right)$. We say that $L$ is generated by the section $\sigma$ over the constraints manifold $C$.

Let $\varsigma: Y \rightarrow N$ be an affine bundle modeled on $N \times \mathbb{R}$ and let $\eta: Y \rightarrow Z$ be an epimorphism of affine bundles such that $\eta(y+r)=\eta(y)+r$ (it is a morphism of principal bundles). We denote by $\underline{\eta}$ the underlying epimorphism $\underline{\eta}: N \rightarrow M$. It follows that for $m \in M$ the subbundle $\eta^{-\overline{1}}\left(Z_{m}\right)$ is trivial (has distinguished constant sections). Let $\sigma$ be a section of $\varsigma$. We say that a point $n \in N$ is critical for $\sigma$ if

$$
\mathrm{d}\left(\left.\sigma\right|_{N_{m}}\right)(n)=\mathrm{d} \sigma_{0}(n)
$$

for a constant section $\sigma_{0}$ of $Y \mid N_{m}$. Let $S(\sigma)$ be the set of critical points of $\sigma$. The condition (13) implies that there exists a section $\sigma^{\prime}$ of $\zeta$ such that $\mathrm{d}_{n}\left(\sigma-\eta^{*} \sigma^{\prime}\right)=0$. We define a mapping

$$
\chi: S \rightarrow \mathrm{P} Z, \quad n \mapsto \mathrm{d}_{m} \sigma^{\prime}
$$


Definition 1. A section $\sigma$ of $\varsigma: Y \rightarrow N$ is an affine Morse family if for a section $\sigma^{\prime}$ of $\zeta: Z \rightarrow M$ the function $\sigma-\eta^{*} \sigma^{\prime}$ on $N$ is a Morse family.

If $\sigma$ is an affine Morse family then $\chi(S)$ is a Lagrangian submanifold of $\left(\mathrm{P} Z, \omega_{\boldsymbol{Z}}\right)$.

3. Special affine spaces and duality. In the category of vector spaces, the dual object is defined as the space of linear functions. In the approach presented here functions are replaced by sections of a bundle. In particular, linear functions on a vector space $\underline{V}$ are replaced by linear sections of a fibration $\zeta: V \rightarrow \underline{V}$ (modeled on $\underline{V} \times \mathbb{R}$ ) in the category of vector spaces. We observe that the space $V^{\ddagger}$ of linear sections is affine, not linear. Morever, an affine section can be uniquely represented by a linear section and a number, i.e. an element of the trivial bundle $V^{\ddagger} \times \mathbb{R}$. This observation leads us to the concept of special affine space (and its model special vector space) which puts a bundle $\zeta: V \rightarrow \underline{V}$ and its dual $V^{\ddagger} \times \mathbb{R}$ into one category.

A special vector space is a pair $\boldsymbol{V}=\left(V, v_{1}\right)$, where $V$ is a vector space and $v_{1} \in V$ is a distinguished, non-zero vector. A special affine space is an affine space modeled on a special vector space. Let $\boldsymbol{V}=\left(V, v_{1}\right)$ and $\boldsymbol{V}^{\prime}=\left(V^{\prime}, v_{1}^{\prime}\right)$ be special vector spaces. A linear mapping $F: V \rightarrow V^{\prime}$ is called a morphism of special vector spaces if $F\left(v_{1}\right)=v_{1}^{\prime}$. A morphism of special affine spaces is an affine mapping such that its linear part is a morphism of special vector spaces. Let $\boldsymbol{A}=\left(A, v_{1}\right)$ be a special affine space with the distinguished vector $v_{1} \in \mathrm{V}(A)$. There is a canonical action of $(\mathbb{R},+)$ on $A$ given by the formula

$$
A \times \mathbb{R} \ni(a, r) \rightarrow a+r v_{1} .
$$

The space of orbits is an affine space modeled on the quotient vector space $\underline{V}=V /\left\{v_{1}\right\}$. We denote it by $\underline{A}$ and the canonical projection $A \rightarrow \underline{A}$ by $\zeta_{A}$. With the action (14) the fibration $\zeta_{\boldsymbol{A}}: A \rightarrow \underline{A}$ is an affine bundle modeled on $\underline{A} \times \mathbb{R}$.

By $\overline{\boldsymbol{A}}$ we denote the special affine space $\left(\bar{A}, v_{1}\right)$. Let $\boldsymbol{A}=\left(A, v_{1}\right)$ and $\boldsymbol{B}=\left(B, w_{1}\right)$ be special affine spaces. The product $A \times B$ is an affine space modeled on $\mathrm{V}(A) \times \mathrm{V}(B)$. Let us denote by $A \otimes B$ the quotient affine space $(A \times B) / L$, where $L \subset \mathrm{V}(A) \times \mathrm{V}(B)$ is a one-dimensional vector subspace spanned by the vector $\left(v_{1},-w_{1}\right)$. We have $\mathrm{V}(A \otimes B)=$ $(\mathrm{V}(A) \times \mathrm{V}(B)) / L$ with a distinguished vector $v_{1} \otimes w_{1}=\left[\left(v_{1}, 0\right)\right]=\left[\left(0, w_{1}\right)\right]$. The product of special affine spaces is the special affine space $\boldsymbol{A} \otimes \boldsymbol{B}=\left(A \otimes B, v_{1} \otimes w_{1}\right)$ modeled on the special vector space $\left(\mathrm{V}(A) \otimes \mathrm{V}(B), v_{1} \otimes w_{1}\right)$, where $\mathrm{V}(A) \times \mathrm{V}(B)=(\mathrm{V}(A) \times \mathrm{V}(B)) / L$

We have obvious isomorphisms

$$
A \otimes B \simeq B \otimes A, \quad A \otimes(B \otimes C) \simeq(A \otimes B) \otimes C .
$$

Since $A$ and $\underline{A}$ are affine spaces, we can distinguish the set of affine sections of $\zeta_{\boldsymbol{A}}$. It is an affine space modeled on the vector space of affine functions on $\underline{A}$ :

$$
\sigma(\underline{a})=\left(\sigma-\sigma^{\prime}\right)(\underline{a}) \cdot v_{1}+\sigma^{\prime}(\underline{a}) .
$$

We consider this space as the dual space to $\boldsymbol{A}$.

In order to interpret the dual object in terms of morphisms, we introduce the distinguished special affine space $\boldsymbol{I}=(\mathbb{R}, 1)$. There is one-to-one correspondence between affine 
sections of the fibration $\zeta_{A}: A \rightarrow \underline{A}$ and morphisms from $\boldsymbol{A}$ to $\boldsymbol{I}$ given by the formula:

$$
\operatorname{Sec}\left(\zeta_{A}\right) \ni \varphi \rightarrow \widetilde{\varphi}: \widetilde{\varphi}(a)=a-\varphi\left(\zeta_{A}(a)\right) .
$$

Let us denote by $A^{\#}$ the set of morphisms $\varphi: A \rightarrow \boldsymbol{I}$. The affine structure on $A^{\#}$ induced from $\operatorname{AffSec} \zeta_{\boldsymbol{A}}$ (see (16)) is modeled on the vector space of affine functions on $\underline{A}$ and is given by

$$
\left(\varphi-\varphi^{\prime}\right)(\underline{a})=\varphi^{\prime}(a)-\varphi(a)
$$

where $\underline{a}=\zeta_{\boldsymbol{A}}(a)$. It follows that the dimensions of $\mathrm{V}(A)$ and $\mathrm{V}\left(A^{\#}\right)$ are equal.

The special affine space $\boldsymbol{A}^{\#}=\left(A^{\#}, \mathbf{1}_{\underline{A}}\right)$, where $\mathbf{1}_{\underline{A}}$ denotes the constant function equal to 1 , will be called the special affine dual to the special affine space $\boldsymbol{A}$.

Example 1 . Let $\underline{A}$ be an affine space. We put $A=\underline{A} \times \mathbb{R}$ with $\mathrm{V}(A)=\mathrm{V}(\underline{A}) \times \mathbb{R}$ and $\boldsymbol{A}=(A,(0,1))$. The dual space $A^{\#}$ is identified with the vector space $\underline{A}^{\dagger}$ of affine functions on $\underline{A}$. With this identification we have $\boldsymbol{A}^{\#}=\left(\underline{A}^{\dagger}, \mathbf{1}_{\underline{A}}\right)$.

REMARK. If we take $\boldsymbol{A}$ as in the example, then $\overline{\boldsymbol{A}}=(\bar{A},(0,1))$ and $\bar{A}=\overline{(\underline{A})} \times \overline{\mathbb{R}}$. Here $\overline{\mathbb{R}}$ denotes the affine space of real numbers with the affine structure dual to the canonical one. The multiplication by -1 gives an isomorphism of $\mathbb{R}$ and $\overline{\mathbb{R}}$ with the identity as the linear part, so we can identify $\overline{\boldsymbol{A}}$ with $(\underline{\bar{A}} \times \mathbb{R},(0,-1))$.

Proposition 1. Let $\boldsymbol{A}=\left(A, v_{1}\right)$ and $\boldsymbol{B}=\left(B, w_{1}\right)$ be special affine spaces. There are the following canonical isomorphisms:

$$
\begin{aligned}
\overline{\overline{\boldsymbol{A}}} & =\boldsymbol{A}, & \overline{\boldsymbol{A}^{\#}} & =(\overline{\boldsymbol{A}})^{\#}, \\
\overline{\boldsymbol{A} \otimes \boldsymbol{B}} & =\overline{\boldsymbol{A}} \otimes \overline{\boldsymbol{B}}, & (\boldsymbol{A} \otimes \boldsymbol{B})^{\#} & =\boldsymbol{A}^{\#} \otimes \boldsymbol{B}^{\#} .
\end{aligned}
$$

Proof. The left-hand identities are obvious. An affine section of $\zeta_{\boldsymbol{A}}$ is also an affine section of $\zeta_{\overline{\boldsymbol{A}}}$ but the affine structures are different. In terms of morphism, $\varphi \in A^{\#}$ gives the same section as $-\varphi \in(\bar{A})^{\#}$.

Now, for each pair $\varphi \in A^{\#}, \psi \in B^{\#}$ we define a mapping

$$
\varphi+\psi: A \times B \rightarrow \mathbb{R}, \quad(a, b) \mapsto \varphi(a)+\psi(b) .
$$

Since

$$
\varphi(a+r v)+\psi(b-r w)=\varphi(a)+\psi(b),
$$

the mapping $\varphi+\psi$ projects to a morphism of special affine spaces $\varphi \oplus \psi: \boldsymbol{A} \otimes \boldsymbol{B} \rightarrow \boldsymbol{I}$. On the other hand, $\varphi \oplus \psi=\varphi^{\prime} \oplus \psi^{\prime}$ if and only if $\varphi(a)+\psi(b)=\varphi^{\prime}(a)+\psi^{\prime}(b)$ for each $a, b$, i.e. if and only if $\varphi-\varphi^{\prime}=\psi^{\prime}-\psi \in \mathbb{R}$. This is equivalent to saying that the pairs $(\varphi, \psi)$ and $\left(\varphi^{\prime}, \psi^{\prime}\right)$ define the same element of $\boldsymbol{A}^{\#} \otimes \boldsymbol{B}^{\#}$.

We have also canonical identifications $\overline{\boldsymbol{I}}=\boldsymbol{I}, \boldsymbol{I} \otimes \boldsymbol{I}=\boldsymbol{I}$ and $\boldsymbol{I}^{\#}=\boldsymbol{I}$ given by the mappings

$$
\begin{aligned}
\mathbb{R} \rightarrow \mathbb{R}, & r \mapsto-r, \\
\mathbb{R} \otimes \mathbb{R} \rightarrow \mathbb{R}, & {[(r, s)] \mapsto r+s, } \\
\mathbb{R}^{\#} \rightarrow \mathbb{R}, & \varphi \mapsto-\varphi(0) .
\end{aligned}
$$


Let $\Psi: \boldsymbol{A} \rightarrow \boldsymbol{B}$ be a morphism of special affine spaces $\boldsymbol{A}=(A, v)$ and $\boldsymbol{B}=(B, w)$. The set

$$
G=\{A \times B \ni(a, b): b=\Psi(b)\}
$$

is invariant with respect to the $\mathbb{R}$-action $((a, b), r) \mapsto(a+r v, a+r w)$ and consequently, it is uniquely determined by its image in $A \otimes \bar{B}$, which is the image of a section of $A \otimes B \rightarrow \underline{A} \times \underline{B}$, defined on the graph $\operatorname{gr}(\underline{\Psi})$ of $\underline{\Psi}: \underline{A} \rightarrow \underline{B}$. The morphism of special affine bundles corresponding to this section,

$$
\operatorname{gr}(\Psi):\left(\zeta_{A} \otimes \zeta_{B}\right)^{-1}(\operatorname{gr}(\underline{\Psi})) \rightarrow I,
$$

will be called the graph of $\Psi$.

Let $\Phi: A \times B \rightarrow \mathbb{R}$ be a bi-affine mapping such that for each $a \in A$ and each $b \in B$ the corresponding mappings

$$
\Phi(a, \cdot): B \rightarrow \mathbb{R}, \quad b^{\prime} \mapsto \Phi\left(a, b^{\prime}\right), \quad \Phi(\cdot, b): A \rightarrow \mathbb{R}, \quad a^{\prime} \mapsto \Phi\left(a^{\prime}, b\right),
$$

are morphisms of special affine spaces. It follows that

$$
\Phi(a, b+w)=\Phi(a+v, b),
$$

i.e. $\Phi$ projects to a mapping $\widetilde{\Phi}: A \otimes B \rightarrow \mathbb{R}$. We say that $\Phi$ is special bi-affine. On the other hand, we say that a mapping

$$
\widetilde{\Phi}: \boldsymbol{A} \otimes \boldsymbol{B} \rightarrow \boldsymbol{I}
$$

is special bi-affine if its pull-back to a function $\Phi: A \times B \rightarrow I$ is special bi-affine. A special bi-affine mapping $\Phi: A \times B \rightarrow \mathbb{R}$ induces mappings

$$
\Phi_{l}: A \rightarrow B^{\#}, \quad a \mapsto \Phi(a, \cdot),
$$

and

$$
\Phi_{r}: B \rightarrow A^{\#}, \quad b \mapsto \Phi(\cdot, b) .
$$

For $r \in \mathbb{R}$ we have $\Phi_{l}(a+r v)(b)=\Phi_{l}(a)(b+r w)=\Phi_{l}(a)(b)+r \mathbf{1}(a)$ and, according to (16), $\Phi_{l}(a+r v)-\Phi_{l}(a)=-r \mathbf{1}$. It follows that $\Phi_{l}$ is a morphism of special affine spaces

$$
\Phi_{l}: \overline{\boldsymbol{A}} \rightarrow \boldsymbol{B}^{\#}
$$

and similarly,

$$
\Phi_{r}: \bar{B} \rightarrow \boldsymbol{A}^{\#}
$$

We say that $\Phi$ is nondegenerate if $\Phi_{l}, \Phi_{r}$ are injective. For injective $\Phi_{l}, \Phi_{r}$, we have

$$
\operatorname{dim} \bigvee(A) \leqslant \operatorname{dim} \bigvee\left(B^{\#}\right)=\operatorname{dim} \bigvee(B) \leqslant \operatorname{dim} \bigvee(A),
$$

i.e. they are isomorphisms. A pairing between special affine spaces $\boldsymbol{A}=\left(A, v_{1}\right)$ and $\boldsymbol{B}=\left(B, w_{1}\right)$ is a nondegenerate special bi-affine mapping

$$
\Phi: \boldsymbol{A} \otimes \overline{\boldsymbol{B}} \rightarrow \boldsymbol{I}
$$

EXAmple 2. Let $\boldsymbol{A}=(A, v)$ be a special affine space. The mapping

$$
\Delta_{A}: A^{\#} \otimes \bar{A} \rightarrow \boldsymbol{I}, \quad(\varphi, a) \mapsto-\varphi(a),
$$

is the canonical pairing between $\boldsymbol{A}^{\#}$ and $\boldsymbol{A}$, while the mapping

$$
\bar{\Delta}_{A}: A \otimes \bar{A}^{\#} \rightarrow \boldsymbol{I}, \quad(a, \varphi) \mapsto \varphi(a),
$$


is the canonical pairing between $\boldsymbol{A}$ and $\boldsymbol{A}^{\#}$. Since these pairings are nondegenerate, we have the canonical isomorphism of $\boldsymbol{A}$ and $\left(\boldsymbol{A}^{\#}\right)^{\#}$.

For a morphism $\Phi: \boldsymbol{A} \rightarrow \boldsymbol{B}$ of special affine spaces, we define the dual morphism $\Phi^{\#}: \boldsymbol{B}^{\#} \rightarrow \boldsymbol{A}^{\#}$ by the formula

$$
\Phi^{\#}(\psi)=\psi \circ \Phi
$$

Proposition 2. Let $\Phi: \boldsymbol{A} \rightarrow \boldsymbol{B}$ be a morphism of special affine spaces. Then

$$
\left(\Phi^{\#}\right)^{\#}=\Phi,
$$

where we have used the canonical isomorphisms $\left(\boldsymbol{A}^{\#}\right)^{\#}=\boldsymbol{A}$ and $\left(\boldsymbol{B}^{\#}\right)^{\#}=\boldsymbol{B}$.

Proof. Let $\chi \in\left(A^{\#}\right)^{\#}$ and $a \in A$ be related by the canonical isomorphism, i.e. let $\chi(\varphi)=-\varphi(a)$ for each $\varphi \in A^{\#}$. By the definition of the dual morphism, we have for each $\psi \in B^{\#}$

$$
\left(\Phi^{\# \#}(\chi)\right)(\psi)=\chi\left(\Phi^{\#}(\psi)\right)=-\left(\Phi^{\#}(\psi)\right)(a)=-\psi(\Phi(a)),
$$

which implies that $\Phi^{\# \#}(\chi)$ and $\Phi(a)$ are related by the canonical isomorphism of $\boldsymbol{B}$ and

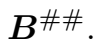

For $\boldsymbol{B}=\boldsymbol{A}^{\#}$ we have $\Phi^{\#}: \boldsymbol{A}^{\# \#}=\boldsymbol{A} \rightarrow \boldsymbol{A}^{\#}$.

Definition 2. A morphism $\Phi: \boldsymbol{A} \rightarrow \boldsymbol{A}^{\#}$ is called self-dual if $\Phi=\Phi^{\#}$.

As we have already noticed, the model vector space for $A^{\#}$ is the vector space of affine functions on $\underline{A}$. The distinguished function is the constant unit function. It follows that the model vector space for $\underline{A^{\#}}$ is the space $\mathrm{V}(A)^{*}$. The canonical projection $A^{\#} \rightarrow \underline{A^{\#}}$ corresponds to extracting from an affine function its linear part.

Proposition 3. Let $\Phi: \boldsymbol{A} \rightarrow \boldsymbol{B}$ be a morphism of special affine spaces. Then $\mathrm{V}\left(\Phi^{\#}\right)=$ $\underline{\Phi}^{*}$, where $\mathrm{V}\left(\Phi^{\#}\right)$ is the linear part of $\Phi^{\#}$.

Proof. Let $a \in A$ and let $f$ be an affine function on $\underline{B}$. Then for $\underline{a}=\zeta_{\boldsymbol{A}}(a)$ and $\psi \in B^{\#}$

$$
\begin{aligned}
\left(\mathrm{V}\left(\Phi^{\#}\right)(f)\right)(\underline{a}) & =\left(\Phi^{\#}(\psi+f)-\Phi^{\#}(\psi)\right)(a)=\left(\Phi^{\#}(\psi)\right)(a)-\left(\Phi^{\#}(\psi+f)\right)(a) \\
& =\psi(\Phi(a))-(\psi(\Phi(a))-f(\underline{\Phi}(\underline{a})))=f(\underline{\Phi}(\underline{a}))=\left(\underline{\Phi}^{*} f\right)(a) .
\end{aligned}
$$

Corollary 1. Let $\Phi: \boldsymbol{A} \rightarrow \boldsymbol{A}^{\#}$ be a self-dual morphism. Then the linear part of the induced mapping of affine spaces $\underline{\Phi}: \underline{A} \rightarrow \underline{A^{\#}}$ is skew self-adjoint.

Proof. We have from the previous proposition that

$$
\mathrm{V}\left(\Phi^{\#}\right)=\mathrm{V}(\Phi)^{*}: \mathrm{V}\left(\Phi^{\# \#}\right) \rightarrow \mathrm{V}\left(\Phi^{\#}\right)
$$

We identify the model space $\mathrm{V}\left(A^{\#}\right)$ with the space $\operatorname{Aff}(\underline{A}, \mathbb{R})$ of affine functions on $\underline{A}$. Similarly, the space $\mathrm{V}\left(A^{\# \#}\right)$ is identified with the space $\operatorname{Aff}\left(\underline{A^{\#}}, \mathbb{R}\right)$. Using the pairing $(28)$ (or $(29)$ ) we see that an element of $\mathrm{V}(A)$ can be interpreted as an affine function on $\underline{A_{\#}^{\#}}$. With this interpretation the sets $\mathrm{V}(A)$ and $\mathrm{V}\left(A^{\# \#}\right)$ are equal and the linear part of the canonical isomorphism $A \rightarrow A^{\# \#}$ is the identity. Let $\varphi$ be an affine function on $A^{\#}$ with linear part $\mathrm{v}(\varphi): \mathrm{V}\left(\underline{A^{\#}}\right)=\mathrm{V}(A)^{*} \rightarrow \mathbb{R}$. The same function, interpreted as an affine function on $\underline{\overline{A^{\#}}}$ has the linear part $-\mathrm{v}(\varphi)$. It follows that the canonical isomorphism of $\boldsymbol{A}$ and $\boldsymbol{A}^{\# \#}$ projects to the minus standard isomorphism of $\mathrm{V}(A)$ and $\mathrm{V}(A)^{* *}$. 
The linear part of a self-dual morphism projects then to a skew self-adjoint linear mapping.

Proposition 4. Let $\Phi: \boldsymbol{A} \rightarrow \boldsymbol{A}^{\#}$ be a morphism of special affine spaces. Then $\Phi$ is self-dual if and only if for each pair $a, b \in A$

$$
\Phi(b)(a)=-\Phi(a)(b) .
$$

Proof. Let $\psi \in A^{\# \#}$ and $b \in A$. By the definition of the dual morphism $\Phi^{\#}(\psi)(b)=$ $\psi(\Phi(b))$. If $\psi$ and $a \in A$ are related by the canonical isomorphism $\boldsymbol{A} \rightarrow \boldsymbol{A}^{\# \#}$ then $\psi(\Phi(b))=-\Phi(b)(a)$ and we get the formula

$$
\Phi^{\#}(a)(b)=-\Phi(b)(a) .
$$

It follows that $\Phi$ is self-dual if and only if $\Phi(a)(b)=-\Phi(b)(a)$ for each pair $a, b \in A$.

Let us choose a point $a_{0} \in A$ and $\varphi_{0} \in A^{\#}$. We define an isomorphism $A \rightarrow V \times \mathbb{R}$, where $V=\mathrm{V}(\underline{A})$, by

$$
A \ni a \mapsto\left(\mathrm{v}\left(\zeta_{A}\right)\left(a-a_{0}\right), \varphi_{0}(a)\right) \in V \times \mathbb{R},
$$

where $\zeta_{A}: A \rightarrow \underline{A}$ is the canonical projection. The affine structure in $V \times \mathbb{R}$ induced by the affine structure of $A$ coincides with the canonical affine structure of a vector space and the distinguished vector is $(0,1)$.

Now, let $\varphi \in A^{\#}$. We have

$$
\varphi(a)=\varphi_{0}(a)-\left(\varphi-\varphi_{0}\right)\left(\zeta_{A}(a)\right)=\varphi_{0}(a)-\mathrm{v}\left(\varphi-\varphi_{0}\right)\left(a-a_{0}\right)-\left(-\varphi\left(a_{0}\right)\right) .
$$

The mapping

$$
A^{\#} \ni \varphi \mapsto\left(\mathrm{v}\left(\varphi-\varphi_{0}\right),-\varphi\left(a_{0}\right)\right) \in V^{*} \times \mathbb{R}
$$

gives an isomorphism of special affine spaces. The affine structure in $V^{*} \times \mathbb{R}$ induced by the affine structure of $A^{\#}$ is the canonical affine structure of a vector space and the distinguished vector is $(0,1)$. With the isomorphisms $(34),(36)$ the pairing $\Delta_{\boldsymbol{A}}$ reads

$$
\Delta_{A}:((f, t),(v, r)) \mapsto t-r+\langle f, v\rangle .
$$

REMARK. We must be aware of the fact that in the formula $(37)$ the pair $(v, r)$ represents an element of $\bar{A}$ and the affine structure in $\bar{A}$ does not correspond to the canonical affine structure on the vector space $V \times \mathbb{R}$ but to the conjugate one. This implies that for given $(f, t)$ the linear part of the mapping $(v, r) \mapsto t-r+\langle f, v\rangle$ is $r-\langle f, v\rangle$.

TheOREM 1. Let $\underline{\Phi}: \underline{A} \rightarrow \underline{A^{\#}}$ be an affine mapping such that its linear part is skew self-adjoint. There exists exactly one lift of $\underline{\Phi}$ to a self-dual morphism $\Phi: \boldsymbol{A} \rightarrow \boldsymbol{A}^{\#}$.

Proof. Let $a_{0} \in A$ and $\varphi \in A^{\#}$ and let $\Phi: \boldsymbol{A} \rightarrow \boldsymbol{A}^{\#}$ be a morphism. With the isomorphisms (34) and (36) we represent $\Phi$ by a quadruple $(F, f, g, t)$, where $F \in \operatorname{Lin}\left(V, V^{*}\right)$, $f, g \in V^{*}, t \in \mathbb{R}:$

$$
\Phi(v, r)=(F(v)+f, g(v)+t+r) .
$$

$\Phi$ is self-dual if and only if (32) is satisfied, i.e.

$$
\Delta_{A}((F(v)+f, g(v)+t+r),(w, s))=-\Delta_{A}((F(w)+f, g(w)+t+s),(v, r))
$$


for any pairs $(v, r),(w, s)$. Using the formula (37) we obtain

$$
\langle F(v)+f, w\rangle+g(v)+t+r-s=-\langle F(w)+f, v\rangle-g(w)-t-s+r .
$$

It follows that $\Phi$ is self-dual if and only if $F=-F^{*}, f=-g$ and $t=0$. This implies that a self-dual morphism is uniquely determined by $F$ and $f$, i.e. by the mapping $\underline{\Phi}: \underline{A} \rightarrow \underline{A^{\#}}$ with the skew self-adjoint linear part.

3.1. Special affine bundles. A special vector bundle is a vector bundle with a distinguished non-vanishing section and a special affine bundle is an affine bundle modeled on a special affine space. A fibre of a special vector bundle is a special vector space and a fibre of a special afffine bundle is a special affine space. Let $\boldsymbol{A}=(A, X)$ and $\boldsymbol{B}=(B, Y)$ be special affine bundles. We define in an obvious way special affine bundles $\overline{\boldsymbol{A}}, \boldsymbol{A} \otimes \boldsymbol{B}$, $\boldsymbol{A}^{\#}$ and $\boldsymbol{A} \otimes_{M} \boldsymbol{B}$ if $A, B$ have the same base manifold $M$. We have also an affine bundle $\underline{A}=A /\{X\}$ and the canonical projection $\zeta_{A}: A \rightarrow \underline{A}$.

Let $\boldsymbol{V}=(V, X)$ and $\boldsymbol{V}^{\prime}=\left(V^{\prime}, X^{\prime}\right)$ be special vector bundles. A morphism $F: V \rightarrow V^{\prime}$ of vector bundles is called a morphism of special vector bundles if $X$ and $X^{\prime}$ are $F$-related. A morphism of special affine bundles is a morphism of affine bundles such that its linear part is a morphism of special vector bundles.

EXAMPLE 3 . Let $\zeta: Z \rightarrow M$ be an affine fibration modeled on the trivial fibration $p r_{M}: M \times \mathbb{R} \rightarrow M$. A pair $\boldsymbol{Z}=\left(Z, \mathbf{1}_{M}\right)$ is a special affine bundle. Also the pair $\mathrm{C} \boldsymbol{Z}=$ $\left(\mathrm{C} Z,\left(0, \mathbf{1}_{M}\right)\right)$ is a special affine fibration with $\underline{\mathrm{C} Z}=\mathrm{P} Z$.

EXAmple 4 . Let $p r_{Z}: Y=Z \times \mathbb{R} \rightarrow Z$ be a trivial affine bundle and let $\boldsymbol{Y}=\left(Y, \mathbf{1}_{Z}\right)$. We define a morphism of special affine bundles

$$
\lambda_{Z}: \boldsymbol{Y} \rightarrow \boldsymbol{Z}, \quad(z, r) \mapsto z+r
$$

which gives a mapping $\lambda_{Z}^{*}: \operatorname{Sec}(Z) \rightarrow \operatorname{Sec}(Y)=\mathcal{C}^{\infty}(Z)$. We have

$$
\lambda_{Z}^{*} \sigma(z)=\sigma(\zeta(z))-z .
$$

In a trivialization provided by a section, we have

$$
\lambda_{Z}^{*} \sigma(m, t)=\sigma(m)-t
$$

The image of the induced relation $\mathrm{P} \lambda_{Z}$ is a coisotropic submanifold $K_{-1}$ of $\mathrm{T}^{*} Z$,

$$
K_{-1}=\left\{a:\left\langle a, X_{1}\right\rangle=-1\right\},
$$

where $X_{1}$ is a vector field on $Z$ represented by the mapping $Z \times \mathbb{R} \ni(z, r) \rightarrow z+r \in Z$.

Proposition 5. Let $\boldsymbol{Z}=\left(Z, \mathbf{1}_{M}\right)$ and $\boldsymbol{Z}^{\prime}=\left(Z^{\prime}, \mathbf{1}_{M^{\prime}}\right)$ be special affine bundles modeled on $M \times \mathbb{R}$ and $M^{\prime} \times \mathbb{R}$ respectively. There are canonical symplectomorphisms

$$
\left(\mathrm{P}\left(\boldsymbol{Z} \otimes \boldsymbol{Z}^{\prime}\right), \omega_{\boldsymbol{Z} \otimes \boldsymbol{Z}^{\prime}}\right)=\left(\mathrm{P} \boldsymbol{Z} \times \mathrm{P} \boldsymbol{Z}^{\prime}, \omega_{\boldsymbol{Z}}+\omega_{\boldsymbol{Z}^{\prime}}\right), \quad\left(\mathrm{P} \bar{Z}, \omega_{\bar{Z}}\right)=\left(\mathrm{P} \boldsymbol{Z},-\omega_{\boldsymbol{Z}}\right) .
$$

Proof. (1) Let $\sigma, \psi$ be sections of $Z$ and $\sigma^{\prime}, \psi^{\prime}$ sections of $Z^{\prime}$. We have from the definition of $Z \otimes Z^{\prime}$ that

$$
\left.\sigma \otimes \sigma^{\prime}-\psi \otimes \psi^{\prime}=(\sigma-\psi)+\left(\sigma^{\prime}-\psi^{\prime}\right)\right) .
$$

It follows that $\left(\sigma \otimes \sigma^{\prime},\left(m, m^{\prime}\right)\right)$ is equivalent to $\left(\psi \otimes \psi^{\prime},\left(n, n^{\prime}\right)\right)$ if and only if $(\sigma, m)$ is equivalent to $(\psi, n)$ and $\left(\sigma^{\prime}, m^{\prime}\right)$ is equivalent to $\left(\psi^{\prime}, n^{\prime}\right)$. The correspondence $\sigma \otimes \sigma^{\prime} \rightarrow$ 
$\left(\mathrm{d} \sigma(m), \mathrm{d} \sigma^{\prime}\left(m^{\prime}\right)\right)$ gives a diffeomorphism of manifolds $\mathrm{P}\left(Z \otimes Z^{\prime}\right)$ and $\mathrm{P} Z \times \mathrm{P} Z^{\prime}$. It is easy to check that this isomorphism is also a symplectomorphism.

(2) It is obvious that $\mathrm{P} Z$ and $\mathrm{P} \bar{Z}$ are equal as manifolds. Let $\sigma$ be a section of $Z$. The same mapping interpreted as a section of $\bar{Z}$ will be denoted by $\bar{\sigma}$. Since $\sigma-\sigma^{\prime}=\bar{\sigma}^{\prime}-\bar{\sigma}$, the isomorphisms $I_{\mathrm{d} \sigma}: \mathrm{P} Z \rightarrow \mathrm{T}^{*} M$ and $I_{\mathrm{d} \bar{\sigma}}: \mathrm{P} \bar{Z} \rightarrow \mathrm{T}^{*} M$ are related by $I_{\mathrm{d} \sigma}=-I_{\mathrm{d} \bar{\sigma}}$. It follows that

$$
\omega_{\bar{Z}}=I_{\mathrm{d} \bar{\sigma}}^{*} \omega_{M}=-I_{\mathrm{d} \sigma}^{*} \omega_{M}=-\omega_{Z} .
$$

We have also similar equalities for contact fibrations:

$$
\mathrm{C} \bar{Z}=\overline{\mathrm{C} Z}, \quad \mathrm{C}\left(Z \otimes Z^{\prime}\right)=\mathrm{C} Z \otimes \mathrm{C} Z^{\prime} .
$$

4. Reduced tangent bundles. Let $\boldsymbol{Z}=\left(Z, \mathbf{1}_{M}\right)$ be, as before, a special affine bundle modeled on the trivial bundle $M \times \mathbb{R}$. We denote by $\phi$ the $\mathbb{R}$-action on $Z: \phi(z, r)=z+r$. The $\mathbb{R}$-action $\phi$ induces an $\mathbb{R}$-action $\phi_{*}$ on $\mathrm{T} Z$. In this section we provide an interpretation of special affine bundles dual to $\mathrm{P} Z \times \mathbb{R}$ and $C Z$ in terms of vectors tangent to $Z$ and the action $\phi$.

We will make use of the correspondence (40) between sections of $\zeta: Z \rightarrow M$ and functions on $Z$. For $\sigma \in \operatorname{Sec}(\zeta)$ we denote by $f_{\sigma}$ the corresponding function on $Z$. For each $v \in \mathrm{T}_{z} Z$ we have a mapping

$$
\mathcal{C}^{\infty}(Z) \ni f \mapsto\left\langle\mathrm{d}_{z} f, v\right\rangle \in \mathbb{R}
$$

and the induced mapping

$$
\operatorname{Sec}(\zeta) \ni \sigma \mapsto\left\langle\mathrm{d}_{z} f_{\sigma}, v\right\rangle \in \mathbb{R}
$$

In a trivialization $f_{\sigma}(m, s)=\sigma(m)-s, v=\underline{v}+\dot{s} \partial / \partial s$, where $\underline{v} \in \mathrm{T}_{\zeta(z)} M$, and the formula (44) takes the form

$$
\sigma \mapsto\left\langle\mathrm{d}_{m} \sigma, \underline{v}\right\rangle-\dot{s} .
$$

This shows that $v$ defines an affine function on $\mathrm{P}_{\zeta(z)} Z$ and the vector $\partial / \partial s$ gives the function $-\mathbf{1}_{\mathrm{P} Z}$. Two vectors $v, v^{\prime}$ define the same function on $\mathrm{P}_{\zeta(z)} Z$ if and only if they are in the same orbit of the $\mathbb{R}$-action $\phi_{*}$. We will denote by $\widetilde{\mathrm{T}} Z$ the space of orbits of this action and we will call it the reduced tangent bundle. It is a vector bundle over $M$ and there is the canonical projection $\widetilde{\mathrm{T}} \zeta: \widetilde{\mathrm{T}} Z \rightarrow \mathrm{T} M$ induced by the tangent projection $\mathrm{T} \zeta: \mathrm{T} Z \rightarrow \mathrm{T} M$ and the reduced canonical projection $\widetilde{\tau}_{Z}: \widetilde{\mathrm{T}} Z \rightarrow M$.

This way we have obtained a bi-affine mapping

$$
\Phi: \mathrm{P} Z \times_{M} \widetilde{\mathrm{T}} Z \rightarrow \mathbb{R}, \quad\left(\mathrm{d}_{\zeta(z)} \sigma,[v]\right) \mapsto\left\langle\mathrm{d}_{z} f_{\sigma}, v\right\rangle \in \mathbb{R}
$$

which extends to a special bi-affine mapping (denoted by the same letter)

$$
\Phi:(\mathrm{P} Z \times \mathbb{R},(0,1)) \bigotimes_{M}\left(\widetilde{\mathrm{T}} Z,-X_{1}\right) \rightarrow \mathbb{R},
$$

where $-X_{1}$ is the fundamental vector field for the action $\phi_{*}$, i.e. in local trivialization $X_{1}=\partial / \partial s$.

The induced mapping $(26) \Phi_{r}: \widetilde{T} Z \rightarrow(\mathrm{P} Z \times \mathbb{R})^{\#}=\mathrm{P} Z^{\dagger}$ (see Example 1) is bijective and defines an isomorphism of special affine bundles

$$
\Phi_{r}: \overline{\left(\widetilde{\mathrm{T}} Z,-X_{1}\right)} \rightarrow(\mathrm{P} Z \times \mathbb{R},(0,1))^{\#}=\left(\mathrm{P} Z^{\dagger}, \mathbf{1}_{\mathrm{P} Z}\right) .
$$


Since $\widetilde{\mathrm{T}} Z$ is a vector bundle, we have an involution $v \mapsto-v$, which is an isomorphism of special vector (affine) spaces

$$
\overline{\left(\widetilde{\mathrm{T}} Z,-X_{1}\right)} \simeq\left(\widetilde{\mathrm{T}} Z, X_{1}\right) .
$$

In the following we identify the special affine dual $(\mathrm{P} Z \times \mathbb{R},(0,1))^{\#}$ with $\widetilde{\mathrm{T}} Z=\left(\widetilde{\mathrm{T}} Z, X_{1}\right)$. In a trivialization $I_{\sigma}$ provided by a section $\sigma_{0}$, we have $Z \simeq M \times \mathbb{R}, \mathrm{P} Z \simeq \mathrm{T}^{*} M$ and $\widetilde{\mathrm{T}} Z \simeq \mathrm{T} M \times \mathbb{R}$, with

$$
(p, 0)(\underline{v}, \dot{s})=\dot{s}-\langle p, \underline{v}\rangle .
$$

REMARK. The trivialization $\widetilde{\mathrm{T}} Z \simeq \mathrm{T} M \times \mathbb{R}$ is given by the decomposition of a vector tangent to $Z$ :

$$
\mathrm{T} Z \ni v=\underline{v}+\dot{s} \frac{\partial}{\partial s}
$$

and it is not compatible with the special affine structure $\left(\widetilde{\mathrm{T}} Z,-X_{1}\right)$. The trivialization $v \mapsto(\underline{v}, r)=(\underline{v},-\dot{s})$ is compatible and gives

$$
(\underline{v}, r)(p, 0)=-(p, 0)(\underline{v}, r)=r+\langle p, \underline{v}\rangle
$$

(see $(28)$ and $(38))$.

REMARK. We can identify the manifolds $\mathrm{P} Z$ and $\mathrm{P} \bar{Z}$ and consequently, the bundles $(\mathrm{P} Z)^{\dagger}$ and $(\mathrm{P} \bar{Z})^{\dagger}$ of affine functions. However, if $v \in \mathrm{T} Z$ defines a function $g_{v}$ on $\mathrm{P} Z$, then the function defined by $v$ on $\mathrm{P} \bar{Z}$ is $-g_{v}$. The natural isomorphism of $\mathrm{P}^{\dagger} Z$ and $\mathrm{P}^{\dagger} \bar{Z}$ corresponds to the multiplication by -1 in $\widetilde{\mathrm{T}} Z$.

For each $v \in \mathrm{T}_{m} M$ there is a unique element $\widetilde{v} \in \widetilde{\mathrm{T}}_{m} Z$ such that $\widetilde{\mathrm{T}} \zeta(\widetilde{v})=v$ and $(p, 0)(\widetilde{v})=0$. In the trivial case this means that $r=\langle p, v\rangle$. This justifies the notation

$$
\widetilde{v}=\langle p, v\rangle
$$

which will be used in the following.

Proposition 6. Let $\boldsymbol{Z}=\left(Z, \mathbf{1}_{M}\right)$ and $\boldsymbol{Z}^{\prime}=\left(Z^{\prime}, \mathbf{1}_{M^{\prime}}\right)$ be special affine bundles modeled on $M \times \mathbb{R}$ and $M^{\prime} \times \mathbb{R}$ respectively. There are canonical isomorphisms

$$
\widetilde{\mathrm{T}} I \simeq I, \quad \widetilde{\mathrm{T}}\left(Z \otimes Z^{\prime}\right) \simeq \widetilde{\mathrm{T}} Z \otimes \widetilde{\mathrm{T}} Z^{\prime} .
$$

Proof. $\mathrm{T} I=\mathbb{R} \times \mathbb{R}$ and $\phi_{*}((s, \dot{s}), r)=(s+r, \dot{s})$. It follows that the space of orbits is parametrized by $\dot{s}$ and the distinguished vector is 1 .

The second isomorphism follows from Proposition 1 and Proposition 5:

$$
\widetilde{\mathrm{T}}\left(Z \otimes Z^{\prime}\right)=\left(\mathrm{P}\left(Z \otimes Z^{\prime}\right)\right)^{\dagger}=\left(\mathrm{P} Z \times \mathrm{P} Z^{\prime}\right)^{\dagger}=\mathrm{P}^{\dagger} Z \otimes \mathrm{P}^{\dagger} Z^{\prime}=\widetilde{\mathrm{T}} Z \otimes \widetilde{\mathrm{T}} Z^{\prime} .
$$

Now, we find the dual to the contact bundle $C Z$. As before, we associate to a vector $v \in \mathrm{T}_{z} Z$ a mapping $\mathcal{C}^{\infty}(Z) \rightarrow \mathbb{R}$, this time by the formula

$$
f \mapsto\left\langle\mathrm{d}_{z} f, v\right\rangle+f
$$

which gives for $f=f_{\sigma}$, in a trivialization provided by a section of $\zeta$,

$$
\operatorname{Sec}(\zeta) \ni \sigma \mapsto\left\langle\mathrm{d}_{m} \sigma, \underline{v}\right\rangle-\dot{s}+\sigma(m)-s
$$

This mapping projects to a special affine mapping $C_{m} Z \rightarrow \mathbb{R}$ and this way a vector $v \in \mathrm{T}_{z} Z$ defines an element of $\mathrm{C}_{m}^{\#} Z=\left(\mathrm{C}_{m} Z\right)^{\#}$, where $m=\zeta(z)$. Thus we have a mapping $\mathrm{T} Z \rightarrow \mathrm{C}^{\#} Z$. It follows from the formula (56) that this mapping is surjective 
and when restricted to $\mathrm{T}_{z} Z$ it is bijective. Two vectors $v \in \mathrm{T}_{z} Z, v^{\prime} \in \mathrm{T}_{z^{\prime}} Z$ define the same element of $C^{\#} Z$ if $\zeta(z)=\zeta\left(z^{\prime}\right)$ and

$$
v^{\prime}=\phi_{*}(v, r)-r X_{1}\left(z^{\prime}\right),
$$

i.e. they are in the same orbit of an $\mathbb{R}$-action on $\mathrm{T} Z$ :

$$
(v, r) \mapsto \phi_{*}(v, r)-r X_{1}(z+r) .
$$

The space of orbits of this action will be denoted by $\overline{\mathrm{T}} Z$. The vector bundle structure of $\mathrm{T} Z$ induces a structure of an affine bundle on $\overline{\mathrm{T}} Z$ with model vector bundle $\widetilde{\mathrm{T}} Z$. We denote by $\overline{\mathrm{T}} \zeta$ and $\bar{\tau}_{\boldsymbol{Z}}$ the induced projections $\overline{\mathrm{T}} Z \rightarrow \mathrm{T} M$ and $\overline{\mathrm{T}} Z \rightarrow M$.

The mapping

$$
((\sigma, m), v) \mapsto\left\langle\mathrm{d}_{z} f_{\sigma}, v\right\rangle+f_{\sigma}(z), \quad \zeta(z)=m,
$$

projects then to a bi-affine mapping

$$
\mathrm{C} Z \times_{M} \overline{\mathrm{T}} Z \rightarrow \mathbb{R}
$$

and a special bi-affine and nondegenerate mapping

$$
\mathrm{C} Z \otimes_{M}\left(\overline{\mathrm{T}} Z,-X_{1}\right) \rightarrow I
$$

which gives an isomorphism

$$
\mathrm{C}^{\#} \boldsymbol{Z} \simeq \overline{\left(\overline{\mathrm{T}} Z,-X_{1}\right)}
$$

The bundle $\overline{\left(\overline{\mathrm{T}} Z,-X_{1}\right)}$ is, by definition, equal to $\left(\overline{\overline{\mathrm{T}} Z},-X_{1}\right)$. This means that we have on $\overline{\mathrm{T}} Z$ an affine structure induced from the affine structure on $\mathrm{T} Z$, conjugate to the canonical one. Using the involution $\widetilde{v} \mapsto-\widetilde{v}$ on $\widetilde{\mathrm{T}} Z$, we get $\left(\overline{\overline{\mathrm{T}}} Z,-X_{1}\right)=\left(\overline{\mathrm{T}} Z, X_{1}\right)$ and the isomorphism

$$
\mathrm{C}^{\#} \boldsymbol{Z} \simeq\left(\overline{\mathrm{T}} Z, X_{1}\right)=\overline{\mathrm{T}} Z .
$$

This isomorphism can be deduced also from Proposition 1, an obvious isomorphism $\mathrm{C} Z=\left(\mathrm{P} Z, \mathbf{1}_{\mathrm{P} Z}\right) \otimes_{M} Z$, and the canonical isomorphism $Z^{\#}=Z$. The last isomorphism is provided by the pairing

$$
Z \bigotimes_{M} \bar{Z} \ni z \otimes z^{\prime} \mapsto z-z^{\prime}
$$

REMARK. The involution $v \mapsto-v$ on $\mathrm{T} Z$ gives a correspondence between the $\mathbb{R}$ action (58) and the $\mathbb{R}$-action given by the formula

$$
(v, r) \mapsto \phi_{*}(v, r)+r X_{1}(z+r) .
$$

Let us denote by $\overline{\mathrm{T}}^{c} Z$ the manifold of orbits of the action (62). It is an affine bundle over $M$ with the affine structure induced by the vector bundle structure on $\mathrm{T} Z$ (over $Z$ ) and isomorphic to the affine bundle $\overline{\mathrm{T}} Z$. The model bundle for $\overline{\mathrm{T}}^{c} Z$ is $\widetilde{\mathrm{T}} Z$. This way we have obtained, via the involution in $\mathrm{T} Z$, an isomorphism of special affine bundles

$$
\overline{\overline{\mathrm{T}} Z} \simeq\left(\overline{\mathrm{T}}^{c} Z,-X_{1}\right) .
$$

For every bundle $Z$ we have a canonical projection $\mu$ : $\mathrm{C} Z \rightarrow Z$ which is a surjective morphism of special affine bundles $\zeta_{\mathrm{C} Z}: \mathrm{C} Z \rightarrow \mathrm{P} Z$ and $\zeta: Z \rightarrow M$. There is also the dual injective morphism

$$
\boldsymbol{Z}=\boldsymbol{Z}^{\#} \rightarrow \mathrm{C}^{\#} \boldsymbol{Z}=\overline{\mathrm{T}} \boldsymbol{Z}
$$


which is the composition of the zero section $Z \rightarrow \mathrm{T} Z$ and the canonical projection $\mathrm{T} Z \rightarrow \overline{\mathrm{T}} Z$.

Proposition 7. Let $\boldsymbol{Z}=\left(Z, \mathbf{1}_{M}\right)$ and $\boldsymbol{Z}^{\prime}=\left(Z^{\prime}, \mathbf{1}_{M^{\prime}}\right)$ be special affine bundles modeled on $M \times \mathbb{R}$ and $M^{\prime} \times \mathbb{R}$ respectively. There are canonical isomorphisms

$$
\overline{\mathrm{T}} I \simeq I, \quad \overline{\mathrm{T}} Z \simeq \overline{\overline{\mathrm{T}}} Z, \quad \overline{\mathrm{T}}\left(Z \otimes Z^{\prime}\right) \simeq \overline{\mathrm{T}} Z \otimes \overline{\mathrm{T}} Z^{\prime} .
$$

Proof. From (64) we have the canonical injection $I \rightarrow \overline{\mathrm{T}} I$ which is an isomorphism. The two remaining isomorphisms follow by the duality from (43) and (19). They can be also obtained directly from the definition of $\overline{\mathrm{T}} Z$ as the quotient of the tangent bundle $\mathrm{T} Z$.

Let $\Phi: Z \rightarrow Z^{\prime}$ be a morphism of special affine bundles. The fundamental vector fields $-X_{1}$ and $-X_{1}^{\prime}$ of the canonical $\mathbb{R}$-actions are $\Phi_{*}$-related, i.e. $X_{1}^{\prime}=\Phi_{*} X_{1}$. It follows that the tangent mapping $\mathrm{T} \Phi: \mathrm{T} Z \rightarrow \mathrm{T} Z^{\prime}$ projects to morphisms of special affine bundles

$$
\widetilde{\mathrm{T}} \Phi: \widetilde{\mathrm{T}} \boldsymbol{Z} \rightarrow \widetilde{\mathrm{T}} \boldsymbol{Z}^{\prime}, \quad \overline{\mathrm{T}} \Phi: \overline{\mathrm{T}} \boldsymbol{Z} \rightarrow \overline{\mathrm{T}} \boldsymbol{Z}^{\prime} .
$$

In particular, we can apply $\widetilde{\mathrm{T}}, \bar{\top}$ to a morphism $\varphi: Z \rightarrow I$. Such a morphism corresponds in a unique way to a section of $\zeta$. Let us recall that this correspondence is given by the condition

$$
\varphi \circ \sigma=0
$$

We denote by $\varphi_{\sigma}$ the morphism corresponding to the section $\sigma$.

Remark. A morphism $\varphi_{\sigma}: Z \rightarrow I$ defines a function $\varphi_{\sigma}: Z \rightarrow \mathbb{R}$ which is related to the already introduced function $f_{\sigma}$ by $\varphi_{\sigma}=-f_{\sigma}$.

The reduced tangent morphism

$$
\widetilde{\mathrm{T}} \varphi: \widetilde{\mathrm{T}} Z \rightarrow \widetilde{\mathrm{T}} I=I
$$

and the morphism

$$
\overline{\mathrm{T}} \varphi: \overline{\mathrm{T}} Z \rightarrow \overline{\mathrm{T}} I=I
$$

(see (54), (65)) correspond to sections of $\widetilde{\mathrm{T}} \zeta: \widetilde{\mathrm{T}} Z \rightarrow \mathrm{T} M$ and $\overline{\mathrm{T}} \zeta: \overline{\mathrm{T}} Z \rightarrow \mathrm{T} M$ respectively. If $\varphi=\varphi_{\sigma}$ then we denote these sections by $\mathrm{d}_{\widetilde{\mathrm{T}}} \sigma$ and $\mathrm{d}_{\overline{\mathrm{T}} Z} \sigma$ respectively, and we call them tangent or complete lifts of the section $\sigma$.

In the trivialization provided by a section of $\zeta, \varphi_{\sigma}(m, s)=s-\sigma(m), \widetilde{\mathrm{T}} Z=\mathrm{T} M \times \mathbb{R}$, $\overline{\mathrm{T}} Z=\mathrm{T} M \times \mathbb{R}$, and

$$
\begin{aligned}
\widetilde{\mathrm{T}} \varphi_{\sigma}(v, \dot{s}) & =\dot{s}-\mathrm{d}_{\boldsymbol{\top}} \sigma(v), & \overline{\mathrm{T}} \varphi_{\sigma}(v, t) & =t-\sigma(m)-\mathrm{d}_{\boldsymbol{\top}} \sigma(v), \\
\mathrm{d}_{\widetilde{\mathrm{T}} Z} \sigma & =\mathrm{d}_{\boldsymbol{\top}} \sigma, & \mathrm{d}_{\overline{\mathrm{T}} Z} \sigma & =\mathrm{d}_{\boldsymbol{\top}} \sigma+\sigma,
\end{aligned}
$$

where $v \in \mathrm{T}_{m} M$ and $\mathrm{d}_{\mathrm{T}}$ is the standard tangent lift of a function on $M$ to a function on $\mathrm{T} M: \mathrm{d}_{\mathrm{\top}} f(v)=\langle\mathrm{d} f, v\rangle($ see $[9,2])$.

5. Canonical isomorphisms. It is known (see [4]) that for a vector bundle $\xi: E \rightarrow M$ there is a canonical isomorphism of $\mathrm{T}^{*} E$ and $\mathrm{T}^{*} E^{*}$. The graph of this isomorphism is a Lagrangian submanifold of $\mathrm{T}^{*}\left(E^{*} \times E\right)$ generated by the evaluation function $E^{*} \times{ }_{M} E \ni$ $(a, f) \mapsto\langle f, a\rangle$. 
In the affine case we replace $E$, or rather $E \times \mathbb{R}$ by a special affine bundle $\boldsymbol{A}=\left(A, X_{A}\right)$ and $E^{*} \times \mathbb{R}$ by $\boldsymbol{A}^{\#}$. We have the pairing (28) between $\boldsymbol{A}^{\#}$ and $\boldsymbol{A}$

$$
\Delta_{\boldsymbol{A}}: \boldsymbol{A}^{\#} \otimes_{M} \overline{\boldsymbol{A}} \rightarrow \boldsymbol{I}, \quad(\varphi, a) \mapsto-\varphi(a),
$$

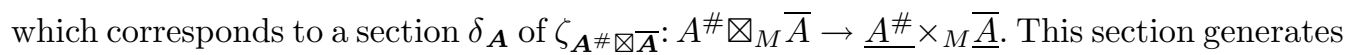
a Lagrangian submanifold of $\mathrm{P}\left(\boldsymbol{A}^{\#} \bigotimes_{M} \overline{\boldsymbol{A}}\right)$ and, together with the canonical embedding

$$
\boldsymbol{A}^{\#} \otimes_{M} \overline{\boldsymbol{A}} \hookrightarrow \boldsymbol{A}^{\#} \otimes \overline{\boldsymbol{A}},
$$

a Lagrangian submanifold $L$ of $\mathrm{P}\left(\boldsymbol{A}^{\#} \otimes \overline{\boldsymbol{A}}\right)=\mathrm{P}\left(\boldsymbol{A}^{\#}\right) \times \mathrm{P}(\overline{\boldsymbol{A}})$. The pull-back of $\delta_{\boldsymbol{A}}$ with respect to the projection

$$
\mathrm{P}\left(A^{\#} \otimes \bar{A}\right) \rightarrow \underline{A^{\#}} \times \underline{\bar{A}}
$$

is a section of

$$
\zeta_{\mathrm{C}\left(A^{\#} \otimes \bar{A}\right)}: \mathrm{C}\left(\boldsymbol{A}^{\#} \otimes \overline{\boldsymbol{A}}\right)=\mathrm{C} A^{\#} \otimes \overline{\mathrm{C} A} \rightarrow \underline{A^{\#}} \times \underline{\bar{A}}
$$

over $L$. This section corresponds to a morphism

$$
\mu_{\boldsymbol{A}}: \zeta_{\mathrm{C}(\boldsymbol{A} \# \otimes \overline{\boldsymbol{A}})}^{-1}(L) \rightarrow I .
$$

TheOREM 2. The morphism $\mu_{\boldsymbol{A}}$ is the graph of an isomorphism $\Psi_{\boldsymbol{A}}: \mathbf{C A} \rightarrow \mathbf{C} \boldsymbol{A}^{\#}$ of special affine bundles. Moreover, $\Psi_{A}$ preserves Liouville forms, i.e.

$$
\theta_{\boldsymbol{A}}=\Psi_{\boldsymbol{A}^{*}}^{*} \theta_{\boldsymbol{A}}
$$

Proof. Let $\psi$ be a section of $\eta: A \rightarrow M$. It defines an isomorphism $I_{\psi}: A \rightarrow E$ of $A$ and the model bundle $E$. It provides also a trivialization of the bundle $\zeta_{A^{\#}}: A^{\#} \rightarrow \underline{A^{\#}}$. Similarly, a section $\psi^{*}$ gives a trivialization of $\zeta_{\boldsymbol{A}}: A \rightarrow \underline{A}$ and an isomorphism of $A^{\#}$ and its model space. Having chosen $\psi$ and $\psi^{*}$, we can identify $A$ with $\underline{E} \times \mathbb{R}$ and $A^{\#}$ with $\underline{E}^{*} \times \mathbb{R}$. We have then

$$
\Delta_{\boldsymbol{A}}((f, t),(e, r))=t-r+\langle f, e\rangle
$$

and

$$
\delta_{\boldsymbol{A}}(f, e)=-\langle f, e\rangle .
$$

According to (42) we identify $\mathrm{P} \overline{\boldsymbol{A}}$ with $\mathrm{P} \boldsymbol{A}$ and the symplectic structure on $\omega_{\overline{\boldsymbol{A}}}$ with $-\omega_{\boldsymbol{A}}$. In the trivialization $\mathrm{P} \boldsymbol{A}=\mathrm{T}^{*} \underline{E}, \mathrm{P} \boldsymbol{A}^{\#}=\mathrm{T}^{*} \underline{E}^{*}, \mathrm{C} \boldsymbol{A}=\mathrm{T}^{*} \underline{E} \times \mathbb{R}$, and $\boldsymbol{C} \boldsymbol{A}^{\#}=\mathrm{T}^{*} \underline{E}^{*} \times \mathbb{R}$.

Let $\left(x^{i}, y^{a}, s\right)$ be an adapted coordinate system on $\underline{E} \times \mathbb{R}$ and $\left(x^{i}, f_{a}, t\right)$ the dual coordinate system on $\underline{E}^{*} \times \mathbb{R}$. Let $\left(x^{i}, y^{a}, p_{j}, \pi_{a}\right)$ be the induced coordinate system on $\mathrm{T}^{*} \underline{E}$ and $\left(x^{i}, f_{a}, q_{i}, \chi^{a}\right)$ the induced coordinate system on $\mathrm{T}^{*} \bar{E}^{*}$. We have

$$
\theta_{\boldsymbol{A}}=p_{i} \mathrm{~d} x^{i}+\pi_{a} \mathrm{~d} y^{a}, \quad-\theta_{\boldsymbol{A}^{\#}}=-q_{i} \mathrm{~d} x^{i}+\chi^{a} \mathrm{~d} f_{a},
$$

and the Lagrangian submanifold $L$, generated by the function $\delta_{\boldsymbol{A}}=-f_{a} y^{a}$ is given by the equations

$$
y^{a}=-\chi^{a}, \quad p_{j}=q_{j}, \quad \pi_{b}=f_{b} .
$$

It follows that $L$ is the graph of a symplectomorphism $\mathrm{T}^{*} \underline{E} \rightarrow \mathrm{T}^{*} \underline{E}^{*}$.

The coordinate systems on $A$ and $A^{\#}$ give the following local expression for $\Delta_{A}$ :

$$
\mu_{\boldsymbol{A}}=t-r+f_{a} y^{a}
$$


and for the corresponding section $\delta_{\boldsymbol{A}}$ :

$$
t-r=-f_{a} y^{a} .
$$

It follows that $\Psi_{\boldsymbol{A}}$ is given in the induced local coordinates by

$$
\begin{array}{rlrl}
x^{i} \circ \Psi_{\boldsymbol{A}} & =x^{i}, & \\
\chi^{a} \circ \Psi_{\boldsymbol{A}} & =-y^{a}, \quad & f_{b} \circ \Psi_{\boldsymbol{A}} & =\pi_{b}, \\
q_{j} \circ \Psi_{\boldsymbol{A}} & =p_{j}, & t \circ \Psi_{\boldsymbol{A}} & =r-f_{a} y^{a} .
\end{array}
$$

It follows from these formulae and from (70) that

$$
\Psi_{\boldsymbol{A}^{*}}^{*} \theta_{\boldsymbol{A}^{\#}}=p_{i} \mathrm{~d} x^{i}-y^{a} \mathrm{~d} \pi_{a}+\mathrm{d}\left(\pi_{a} y^{a}\right)=p_{i} \mathrm{~d} x^{i}+\pi_{a} \mathrm{~d} y^{a}=\theta_{\boldsymbol{A}} .
$$

There are canonical contact structures on the contact fibration $\mathrm{C} \zeta_{A}$ : $\mathrm{C} A \rightarrow \underline{A}$ and on the contact fibration $C \zeta_{A^{\#}}: C A^{\#} \rightarrow \underline{A}^{\#}$. It follows from the theorem that there are also fibrations $\mathrm{C} A \rightarrow \underline{A}^{\#}$ and $\mathrm{C} A^{\#} \rightarrow \underline{A}$, which are special affine bundles. Moreover, we have morphisms (10) of special affine bundles:

$$
\begin{array}{ll}
\mathrm{CA} \rightarrow \boldsymbol{A}, & \mathrm{C} \boldsymbol{A}^{\#} \rightarrow \boldsymbol{A}^{\#}, \\
\mathrm{CA} \rightarrow \boldsymbol{A}^{\#}, & \mathrm{C} \boldsymbol{A}^{\#} \rightarrow \boldsymbol{A} .
\end{array}
$$

The projections $\mathrm{C} \boldsymbol{A} \rightarrow \boldsymbol{A}^{\#}, \mathrm{C} \boldsymbol{A}^{\#} \rightarrow \boldsymbol{A}$, and the related structures can be obtained directly, i.e. without using the mapping $\Psi_{\boldsymbol{A}}$. As an example we give an alternative definition of the projection $\mathrm{C} \boldsymbol{A} \rightarrow \boldsymbol{A}^{\#}$.

First, we define a mapping

$$
\chi: A \times_{M} A \rightarrow \mathrm{T} A
$$

where $\chi(a, b)$ is the vector represented by the curve $\gamma_{a, b}: t \mapsto a+t(b-a)$. In the case under consideration the action $\phi$ of (62) reads

$$
\phi(a, r)=a+r X_{A}(\eta(a))
$$

and the mapping $(\chi(a, b), r) \mapsto \chi\left(a+r X_{A}(\eta(a))\right)$ coincides with the action (62). Hence the mapping $\chi$ projects to

$$
\bar{\chi}: \underline{A} \times_{M} A \rightarrow \overline{\mathrm{T}} A
$$

and for each $\underline{a} \in \underline{A}$ the mapping $\bar{\chi}(\underline{a}, \cdot)$ is a morphism of special affine bundles

$$
\bar{\chi}(\underline{a}, \cdot): \boldsymbol{A} \rightarrow \overline{\mathrm{T}} \boldsymbol{A} \text {. }
$$

The dual morphisms

$$
\bar{\chi}(\underline{a}, \cdot)^{\#}: \mathrm{C}_{\underline{a}} \boldsymbol{A} \rightarrow \boldsymbol{A}^{\#}
$$

define the projection

$$
\mathrm{C} \boldsymbol{A} \rightarrow \boldsymbol{A}^{\#}
$$

5.1. Tangent affine bundles. Let $\xi: E \rightarrow M$ be a vector bundle. It is well known that the tangent manifold $\mathrm{T} E$ carries in a natural way two different structures of a vector bundle: one on the canonical fibration $\tau_{E}: \mathrm{T} E \rightarrow E$ and the second on the tangent fibration $\mathrm{T} \xi: \mathrm{T} E \rightarrow \mathrm{T} M$.

Let $\eta: A \rightarrow M$ be an affine bundle modeled on $\xi: E \rightarrow M$. The tangent manifold $\mathrm{T} A$ is a vector bundle $\tau_{A}$ : T $A \rightarrow A$ and it is also an affine bundle with respect to the tangent fibration $\mathrm{T} \eta: \mathrm{T} A \rightarrow \mathrm{T} M$. The model vector bundle is $\mathrm{T} \xi: \mathrm{T} E \rightarrow \mathrm{T} M$ and the 
affine structure is obtained by applying the tangent functor to mappings which define the affine structure of $A$.

Let $\boldsymbol{A}=\left(A, X_{A}\right)$ be a special affine bundle. The canonical projections

$$
\widetilde{\mathrm{T}} A \rightarrow \mathrm{T} \underline{A} \rightarrow \mathrm{T} M
$$

give a fibration

$$
\widetilde{\mathrm{T}} \eta: \widetilde{\mathrm{T}} A \rightarrow \mathrm{T} M
$$

and the affine bundle structure on $\mathrm{T} A \rightarrow \mathrm{T} M$ induces an affine bundle structure on this fibration. The model vector bundle is the reduced tangent bundle $\widetilde{\mathrm{T}} \xi: \widetilde{\mathrm{T}} E \rightarrow \mathrm{T} M$. To avoid ambiguities we denote by $\dot{+}$ the operation of addition for this structure. The section $X_{A}$ can be lifted to a section $\widetilde{X}_{A}$ of $\widetilde{\mathrm{T}}_{\eta}$ in the following way: for $v \in \mathrm{T}_{m} M$ the vector $\widetilde{X}_{A}(v)$ is the equivalence class of a vector $\widehat{X}_{A}(v)$ in $\mathrm{T}_{0}(m) E$ defined by the formula

$$
\widehat{X}_{A}(v)=\mathrm{v}_{\mathrm{T}} X_{A}\left(0_{E}(m)\right)+\mathrm{T} 0_{E}(v),
$$

where $\mathrm{v}_{\top} X_{A}$ is the standard vertical lift of $X_{A}$ and $0_{E}: M \rightarrow E$ is the zero section of $\eta$.

The pair $\left(\widetilde{\mathrm{T}} A, \widetilde{X}_{A}\right)$ is a special affine bundle over $\mathrm{T} M$. Thus the reduced tangent manifold $\widetilde{\mathrm{T}} A$ carries two special affine structures: with respect to the canonical projection $\widetilde{\tau}_{A}: \widetilde{\top} A \rightarrow \underline{A}$ (the distinguished vector field is the vertical lift of $X_{A}$ ) and with respect to the reduced tangent projection $\widetilde{\mathrm{T}} \eta: \widetilde{\mathrm{T}} A \rightarrow \mathrm{T} M$.

In local coordinates $\left(x^{i}, y^{a}, s\right)$ on $A$ and $\left(x^{i}, y^{a}, \dot{x}^{j}, \dot{y}^{b}, \dot{s}\right)$ on $\widetilde{\mathrm{T}} A$, we have $y^{a} \circ X_{A}=$ $0, s \circ X_{A}=1$ and

$$
\begin{gathered}
y^{a} \circ \widetilde{X}_{A}=0, \dot{y}^{b} \circ \widetilde{X}_{A}=0, \dot{s} \circ \widetilde{X}_{A}=1 \\
\dot{x}_{i} \circ \mathrm{v}_{\mathrm{T}} X_{A}=0, \dot{y}^{a} \circ \mathrm{v}_{\mathrm{T}} X_{A}=0, \dot{s} \circ \mathrm{v}_{\mathrm{T}} X_{A}=1 .
\end{gathered}
$$

It follows that the induced $\mathbb{R}$-actions $\widetilde{\mathrm{T}} A \ni v \mapsto v \dot{+} r \widetilde{X}_{A}$ and $\widetilde{\mathrm{T}} A \ni v \rightarrow v+\mathrm{v}_{\mathrm{\top}} X_{A}$ coincide and the quotient manifold for these actions is $\mathrm{T} \underline{A}$.

The special affine dual to $\left(\widetilde{\mathrm{T}} A, \mathrm{v}_{\mathrm{T}} X_{A}\right)$ is $\mathrm{P} \boldsymbol{A} \times \mathbb{R}$. We show that the special affine dual to $\left(\widetilde{\mathrm{T}} A, \widetilde{X}_{A}\right)$ is $\left(\widetilde{\mathrm{T}} A^{\#}, \widetilde{X}_{A^{\#}}\right)$.

Let us notice first that the equality $\widetilde{\mathrm{T}}(A \otimes B)=\widetilde{\mathrm{T}} A \otimes \widetilde{\mathrm{T}} B$ implies not only the equality

$$
\left(\widetilde{\mathrm{T}}(A \otimes B), \mathrm{v}_{\mathrm{\top}} X_{A \otimes B}\right)=\left(\widetilde{\mathrm{T}} A, \mathrm{v}_{\mathrm{\top}} X_{A}\right) \otimes\left(\widetilde{\mathrm{T}} B, \mathrm{v}_{\mathrm{\top}} X_{B}\right)
$$

which follows from Proposition 6 , but also

$$
\left(\widetilde{\mathrm{T}}(A \otimes B), \widetilde{X}_{A \bigotimes B}\right)=\left(\widetilde{\mathrm{T}} A, \widetilde{X}_{A}\right) \otimes\left(\widetilde{\mathrm{T}} B, \widetilde{X}_{B}\right) .
$$

If $A$ and $B$ have the same base manifold $M$ then (82) implies

$$
\left(\widetilde{\mathrm{T}}\left(A \otimes_{M} B\right), \widetilde{X}_{A \bigotimes B}\right)=\left(\widetilde{\mathrm{T}} A, \widetilde{X}_{A}\right) \bigotimes_{\mathrm{T} M}\left(\widetilde{\mathrm{T}} B, \widetilde{X}_{B}\right) .
$$

The tangent lift $\widetilde{\mathrm{T}} \Delta_{A}$ of the pairing $\Delta_{A}: \boldsymbol{A}^{\#} \otimes \overline{\boldsymbol{A}} \rightarrow I$ is a morphism

$$
\widetilde{\mathrm{T}} \Delta_{A}: \widetilde{\mathrm{T}}\left(\boldsymbol{A}^{\#} \otimes_{M} \overline{\boldsymbol{A}}\right) \rightarrow I,
$$

in local coordinates (see (67) and (74))

$$
\left(\left(x^{i}, y^{a}, \dot{x}^{j}, \dot{y}^{b}, \dot{s}\right),\left(x^{i}, f_{a}, \dot{x}^{j}, \dot{f}_{b}, \dot{t}\right)\right) \mapsto \dot{t}-\dot{s}+\dot{f}_{a} y^{a}+f_{a} \dot{y}^{a} .
$$

It follows from this formula that $\widetilde{\mathrm{T}} \Delta_{A}$ is a pairing

$$
\widetilde{\mathrm{T}} \Delta_{A}:\left(\widetilde{\mathrm{T}} A, \widetilde{X}_{A}\right) \otimes_{\mathrm{T} M} \overline{\left(\widetilde{\mathrm{T}} A^{\#}, \widetilde{X}_{A^{\#}}\right)} \rightarrow I
$$


and from (25) we get an isomorphism

$$
\left(\widetilde{\mathrm{T}} A, \widetilde{X}_{A}\right)^{\#} \simeq\left(\widetilde{\mathrm{T}} A^{\#}, \widetilde{X}_{A^{\#}}\right) .
$$

The same procedure can be applied to the tangent bundle $\overline{\mathrm{T}} A$ and we get two special affine structures on $\overline{\mathrm{T}} A$ : with respect to the canonical projection $\overline{\mathrm{T}} A \rightarrow \underline{A}$ and the tangent projection $\overline{\mathrm{T}} A \rightarrow \mathrm{T} M$.

5.2. The isomorphism $\alpha_{\boldsymbol{Z}}$. Let $\boldsymbol{Z}$ be as in the previous sections. The tangent bundles $\widetilde{\mathrm{T}} Z$ and $\overline{\mathrm{T}} Z$ have been defined as reductions of $\mathrm{T} Z$. This implies that the iterated tangent manifolds $\widetilde{\mathrm{T}} \widetilde{\mathrm{T}} Z, \widetilde{\mathrm{T}} \overline{\mathrm{T}} Z$ and $\overline{\mathrm{T}} \widetilde{\mathrm{T}} Z$ are reductions of the iterated tangent manifold $\mathrm{TT} Z$.

THEOREM 3. The canonical flip $\kappa_{Z}$

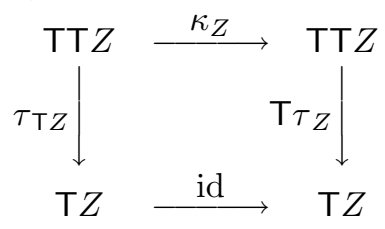

projects to $\widetilde{\kappa}_{Z}$ :

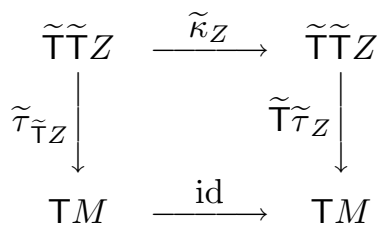

and to $\bar{\kappa}_{Z}$ :

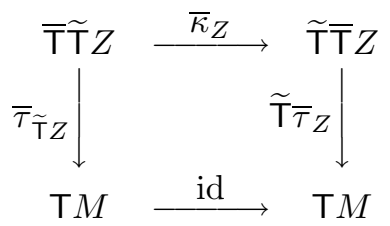

Proof. The canonical $\mathbb{R}$-action $\phi$ on $Z$ induces the tangent action of $T \mathbb{R}=\mathbb{R}^{2}$ on $\mathrm{T} Z$ and the iterated tangent action of $T T \mathbb{R}$ on $T T Z$. Since $\kappa$ is a natural transformation of functors, we have

$$
\mathrm{TT} \phi \circ\left(\kappa_{Z} \times \kappa_{\mathbb{R}}\right)=\kappa_{Z} \circ \mathrm{TT} \phi .
$$

The manifold $\widetilde{\mathrm{T}} Z$ is the manifold of orbits of the kernel subgroup of the homomorphism $\mathrm{T} \mathbb{R} \rightarrow \mathbb{R},(r, \dot{r}) \mapsto \dot{r}$. Similarly, $\overline{\mathrm{T}} Z$ is the manifold of orbits of the kernel of the homomorphism $\mathrm{T} \mathbb{R} \rightarrow \mathbb{R},(r, \dot{r}) \mapsto r+\dot{r}$. The canonical $\mathbb{R}$-actions are induced by these homomorphisms. It follows that $\mathrm{TT} Z$ is $\mathrm{TT} Z$ reduced by the tangent group homomorphism

$$
\mathrm{TT} \mathbb{R} \rightarrow \mathrm{T} \mathbb{R}, \quad\left(r, \dot{r}, r^{\prime}, \dot{r}^{\prime}\right) \mapsto\left(\dot{r}, \dot{r}^{\prime}\right),
$$

and $\widetilde{\mathrm{TT}} Z$ is TT $Z$ reduced by the group homomorphism

$$
\chi_{1}: \mathrm{TT} \mathbb{R} \rightarrow \mathbb{R}, \quad\left(r, \dot{r}, r^{\prime}, \dot{r}^{\prime}\right) \mapsto \dot{r}^{\prime} .
$$

In the same way we interpret $\overline{\mathrm{T}} \widetilde{\mathrm{T}} Z$ as $\mathrm{TT} Z$ reduced by the group homomorphism

$$
\chi_{2}: \mathrm{TT} \mathbb{R} \rightarrow \mathbb{R}, \quad\left(r, \dot{r}, r^{\prime}, \dot{r}^{\prime}\right) \mapsto \dot{r}^{\prime}+\dot{r},
$$


and $\widetilde{\mathrm{T}} \overline{\mathrm{T}} Z$ as TT $Z$ reduced by the group homomorphism

$$
\chi_{3}: \mathrm{TT} \mathbb{R} \rightarrow \mathbb{R}, \quad\left(r, \dot{r}, r^{\prime}, \dot{r}^{\prime}\right) \mapsto \dot{r}^{\prime}+r^{\prime} .
$$

We see that $\chi_{1} \circ \kappa_{Z}=\chi_{1}, \chi_{2} \circ \kappa_{Z}=\chi_{3}$, and consequently $\kappa_{Z}$ projects to diffeomorphisms

$$
\widetilde{\kappa}_{Z}: \widetilde{\mathrm{T}} \widetilde{\mathrm{T}} Z \rightarrow \widetilde{\mathrm{T}} \widetilde{\mathrm{T}} Z, \quad \bar{\kappa}_{Z}: \overline{\mathrm{T}} \widetilde{\mathrm{T}} Z \rightarrow \widetilde{\mathrm{T}} \overline{\mathrm{T}} Z .
$$

The affine structures of $\widetilde{\mathrm{T}} \widetilde{\mathrm{T}} Z, \widetilde{\mathrm{T}} \overline{\mathrm{T}} Z$ and $\overline{\mathrm{T}} \widetilde{\mathrm{T}} Z$ are obtained by reductions from the vector bundle structures of TT $Z$. It follows that $\widetilde{\kappa}_{Z}$ and $\bar{\kappa}_{Z}$ are isomorphisms of the corresponding special affine bundles.

THEOREM 4. There are canonical isomorphisms

$$
\widetilde{\alpha}_{\boldsymbol{Z}}: \widetilde{\mathrm{T}}^{\#} \boldsymbol{Z} \rightarrow \widetilde{\mathrm{T}}^{\#} \widetilde{\mathrm{T}} \boldsymbol{Z} \text { dual to } \widetilde{\kappa}_{\boldsymbol{Z}}
$$

such that the diagram

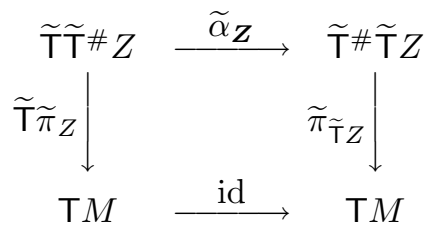

is commutative and

$$
\bar{\alpha}_{Z}: \widetilde{\mathrm{T}} \mathrm{C} \boldsymbol{Z} \rightarrow \mathrm{C} \widetilde{\mathrm{T}} \boldsymbol{Z}
$$

with the commutative diagram

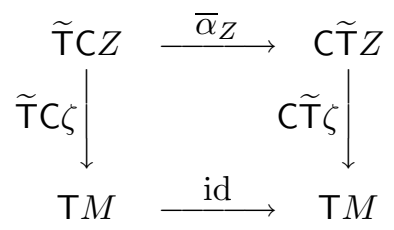

Proof. Follows directly from the previous theorem and from (86).

Both isomorphisms $\widetilde{\alpha}_{\boldsymbol{Z}}$ and $\bar{\alpha}_{\boldsymbol{Z}}$ project to the same diffeomorphism

$$
\underline{\alpha}_{Z}: \mathrm{TP} Z \rightarrow \mathrm{PT} Z \text {. }
$$

Let $\varphi: M \rightarrow \mathrm{P} Z$ be an affine 1 -form. Then $\underline{\alpha}_{Z} \circ \mathrm{T} \varphi: \mathrm{T} M \rightarrow \mathrm{P} \widetilde{\mathrm{T}} Z$ is an affine 1 -form on $\mathrm{T} M$. We call it the complete or tangent lift of $\varphi$ and we will denote it by $\mathrm{d}_{\widetilde{\mathrm{T}}} \varphi$. As in the classical case we have for a section $\sigma$ of $\zeta$ and for an affine 1-form $\varphi$

$$
\mathrm{d}_{\widetilde{\top} \boldsymbol{Z}} \mathrm{d} \sigma=\mathrm{dd}_{\widetilde{\top} \boldsymbol{Z}} \sigma, \quad \mathrm{d}_{\boldsymbol{\top}} \mathrm{d} \varphi=\mathrm{dd}_{\widetilde{\top} \boldsymbol{Z}} \varphi .
$$

PROPOSITION 8.

$$
\bar{\alpha}_{Z}^{*} \theta_{\widetilde{\mathrm{T}} \boldsymbol{Z}}=\mathrm{d}_{\widetilde{\mathrm{T}} \boldsymbol{Z}} \theta_{\boldsymbol{Z}}, \quad \underline{\alpha}_{Z}^{*} \omega_{\widetilde{\mathrm{T}} \boldsymbol{Z}}=\mathrm{d}_{\mathrm{T}} \omega_{\boldsymbol{Z}} .
$$

Proof. In a trivialization given by a section of $\zeta, \widetilde{\mathrm{T}} \mathrm{C} Z \simeq \mathrm{TT}^{*} M \times \mathbb{R}, \mathrm{C} \widetilde{\mathrm{T}} Z \simeq \mathrm{T}^{*} \mathrm{~T} M \times \mathbb{R}$ and $\widetilde{\alpha}_{\boldsymbol{Z}}$ is the trivial lift of $\alpha_{M}$. The first equality follows from the well known equality $\alpha_{M}^{*} \theta_{\mathrm{T} M}=\mathrm{d}_{\mathrm{T}} \theta_{M}$. The second equality is a consequence of the first one and of (96).

6. The dynamics of a charged particle [14]. In gauge theories potentials are interpreted as connections on principal bundles. In electrodynamics the gauge group is 
$(\mathbb{R},+)$ and a potential is a connection on a principal bundle $\zeta: Z \rightarrow M$ over the space-time $M$. The bundle $Z$ can be considered a special affine bundle modeled on $M \times \mathbb{R}$ with the distnguished section $\mathbf{1}_{M}$. An electromagnetic potential is a section $A: M \rightarrow \mathrm{P} Z$.

According to [18] the phase manifold for a particle with charge $e \in \mathbb{R}$ is obtained by the symplectic reduction of $\mathrm{T}^{*} Z$ with respect to the coisotropic submanifold

$$
K_{e}=\left\{p \in \mathrm{T}^{*} Z:\left\langle p, X_{1}\right\rangle=e\right\} .
$$

Let us denote by $\mathrm{P}_{e} Z$ the reduced phase space. It is easy to see that it is an affine bundle modeled on $\mathrm{T}^{*} M$. We show that $\mathrm{P}_{e} Z$ is the phase bundle for a certain special affine bundle $\boldsymbol{Z}_{e}$.

First, let $Y=Z \times \mathbb{R}$ and $\boldsymbol{Y}=\left(Y, \mathbf{1}_{Z}\right)$ as in Example 4. We define an $\mathbb{R}$-action on $Y$ by the formula

$$
(Z \times \mathbb{R}) \times \mathbb{R} \ni((z, r), t) \mapsto(z+t, r+t e) \in Z \times \mathbb{R}=Y .
$$

The space of orbits is an affine bundle modeled on $M \times \mathbb{R}$ and denoted by $Z_{e}$. We denote by $\zeta_{e}$ the canonical projection $Z_{e} \rightarrow M$. The distinguished section of $\mathrm{V}(Y)$ (the function $\mathbf{1}_{Z}$ ) projects to the constant function $\mathbf{1}_{M}$ and the canonical projection $\lambda_{e}: Y \rightarrow Z_{e}$ is a morphism of special affine bundles $\boldsymbol{Y} \rightarrow \boldsymbol{Z}_{e}=\left(Z_{e}, \mathbf{1}_{M}\right)$. The induced $\mathbb{R}$-action on $Z_{e}$ has the form

$$
\lambda_{e}(z, r)+s=\lambda_{e}(z, r+s)=\lambda_{e}(z+t, r+s+t e)
$$

For $e=0$ the bundle $Z_{e}$ is trivial: $Z_{0}=M \times \mathbb{R}$ and for $e \neq 0$ we have a diffeomorphism

$$
\Phi_{e}: Z \rightarrow Z_{e}, \quad z \mapsto \lambda_{e}(z, 0)
$$

The diffeomorphism $\Phi_{e}$ is not a morphism of special affine bundles:

$$
\Phi_{e}(z+r)=\lambda_{e}(z+r, 0)=\lambda_{e}(z,-e r)=\lambda_{e}(z, 0)-e r=\Phi_{e}(z)-e r,
$$

unless $e=-1$ (Example 4).

Let $\sigma$ be a section of $\zeta_{e}$. The function $\lambda_{e}^{*} \sigma$ on $Z$ has the property

$$
X_{1}\left(\lambda_{e}^{*} \sigma\right)=e .
$$

We conclude that the relation $\mathrm{P} Y \rightarrow \mathrm{P} Z_{e}$ induced by $\lambda_{e}$ is the symplectic reduction with respect to the coisotropic submanifold

$$
K_{e}=\left\{p \in \mathrm{T}^{*} Z:\left\langle p, X_{1}\right\rangle=e\right\} .
$$

Thus we have proved

Proposition 9. The phase manifold $P_{e} Z$ for a particle with charge $e$ is the phase bundle for the special affine bundle $\boldsymbol{Z}_{e}$.

The diffeomorphism $\Phi_{e}$ gives a one-to-one correspondence between sections of $\zeta$ and sections of $\zeta_{e}$, for $e \neq 0$. It follows that a chosen section of $\zeta$ provides a trivialization of $Z$ and also of $Z_{e}$. In such trivializations, a section $\sigma$ of $\zeta$ and the corresponding section $\Phi_{e} \circ \sigma$ of $\zeta_{e}$ are functions on $M$ related by the formula

$$
\Phi_{e} \circ \sigma(m)=-e \sigma(m) .
$$

The correspondence $\sigma \rightarrow \Phi_{e} \circ \sigma$ of sections projects to a correspondence of affine covectors and consequently gives a correspondence of affine 1-forms. Let $A$ be a section of $\mathrm{P} \zeta$ and 
$A_{e}$ the corresponding section $\mathrm{P} \zeta_{e}$. In given trivializations, the sections $A$ and $A_{e}$ are 1 -forms related by the formula

$$
A_{e}=-e A \text {. }
$$

The Lagrangian of a charged particle is a section $L_{e}$ of the bundle $\widetilde{\mathrm{T}} \zeta_{a}: \widetilde{\mathrm{T}} Z_{e} \rightarrow \mathrm{T} M$ over the open set $C=\{v \in \mathrm{T} M: g(v, v)>0\}$ and is given by the formula

$$
L(v)=\left\langle A_{e}, v\right\rangle+m \sqrt{g(v, v)}
$$

where $g$ is the metric tensor, $m$ is the mass of the particle, and $\langle$,$\rangle has been defined$ in (53). The Lagrangian section $L_{e}$ generates a Lagrangian submanifold $D_{l, e}$ of $\mathrm{P} Z_{e}$.

The dynamics of the system is the Lagrangian submanifold $D_{e}$ of TP $Z_{e}$

$$
D_{e}=\underline{\alpha}_{Z_{e}}^{-1}\left(D_{l, e}\right) .
$$

We have

$$
D_{e}=\left\{w \in \operatorname{TP} Z_{e}: v=\operatorname{TP} \zeta_{Z_{e}}(w) \in C \text { and } m v=\sqrt{g(v, v)} g\left(p-A_{e}(m)\right)\right\},
$$

where $p=\tau_{\mathrm{P} Z_{e}}(w)$ and $m=\mathrm{P} \zeta_{e}(p)$.

In the Hamiltonian formulation of the dynamics, the generating object is a Morse family $F: N \rightarrow \mathbb{R}$ defined on $N=\mathrm{T} M \times_{M} \mathrm{P} Z$ with the canonical projection

$$
\varsigma: N=\mathrm{T} M \times{ }_{M} \mathrm{P} Z \rightarrow \mathrm{P} Z
$$

by

$$
F(v, p)=L(v)-\langle p, v\rangle .
$$

\section{References}

[1] S. Benenti, Fibrés affines canoniques et mécanique newtonienne, Lyon, 26-30 mai, 1986, Séminaire Sud-Rhodanien de Géométrie, Journées S.M.F.

[2] J. Grabowski and P. Urbański, Tangent lifts of Poisson and related structures, J. Phys. A 28 (1995), 6743-6777.

[3] K. Konieczna, Affine formulation of the Newtonian analytical mechanics, thesis, University of Warsaw, 1995 (in Polish).

[4] K. Konieczna and P. Urbański, Double vector bundles and duality, Arch. Math. 35 (1999), 59-95.

[5] E. Martínez, T. Mestdag, and W. Sarlet, Lie algebroid structures and Lagrangian systems on affine bundles, math.DG/0203178.

[6] E. Massa, E. Pagani, and P. Lorenzoni, On the gauge structure of classical mechanics, Transport Theory Stat. Phys. 29 (2000), 69-91.

[7] E. Massa, S. Vignolo, and D. Bruno, Non-holonomic Lagrangian and Hamiltonian mechanics: an intrinsic approach, J. Phys. A (2002), 6713-6742.

[8] M. R. Menzio and W. Tulczyjew, Infinitesimal symplectic relations and generalized Hamiltonian dynamics, Ann. Inst. H. Poincaré 28 (1978), 349-367.

[9] G. Pidello and W. Tulczyjew, Derivations of differential forms on jet bundles, Ann. Mat. Pura Appl. 147 (1987), 249-265.

[10] W. Sarlet, T. Mestdag, and E. Martínez, Lie algebroid structures on a class of affine bundles, math.DG/0201264. 
[11] W. Sarlet, T. Mestdag, and E. Martínez, Lagrangian equations on affine Lie algebroids, in: Differential Geometry and its Applications, Proc. 8th Int. Conf. (Opava 2001), D. Krupka et al. (eds.) (to appear).

[12] W. Tulczyjew, Hamiltonian systems, Lagrangian systems, and the Legendre transformation, Symposia Math. 14 (1974), 101-114

[13] W. M. Tulczyjew, Frame independence of analytical mechanics, Atti Accad. Sci. Torino 119 (1985).

[14] W. M. Tulczyjew and P. Urbański, An affine framework for the dynamics of charged particles, Atti Accad. Sci. Torino Suppl. 16 (1992), 57-265.

[15] W. M. Tulczyjew, P. Urbański, and S. Zakrzewski, A pseudocategory of principal bundles, Atti Accad. Sci. Torino 122 (1988), 66-72.

[16] P. Urbański, Affine Poisson structure in analytical mechanics, in: Quantization and Infinite-Dimensional Systems, J.-P. Antoine et al. (eds.), Plenum Press, New York and London, 1994, 123-129.

[17] S. Vignolo, A new presymplectic geometrical framework for time-dependent Lagrangian systems, J. Phys. A. 33 (2000), 5117-5135.

[18] A. Weinstein, A universal phase space for particles in Yang-Mills fields, Lett. Math. Phys. 2 (1978), 417-420. 